Published in "IEEE Circuits and Systems Magazine 6(3): 67 - 85 , 2008"

which should be cited to refer to this work.

\title{
Collective Behavior Coordination with Predictive Mechanisms
}

\author{
Hai-Tao Zhang, Michael ZhiQiang Chen, Guy-Bart Stan, Tao Zhou, and Jan M. Maciejowski
}

In natural flocks/swarms, it is very appealing that low-level individual intelligence and communication can yield advanced coordinated collective behaviors such as congregation, synchronization and migration. In the past few years, the discovery of collective flocking behaviors has stimulated much interest in the study of the underlying organizing principles of abundant natural groups, which has led to dramatic advances in this emerging and active research field. Inspired by previous investigations on the predictive intelligence of animals, insects and microorganisms, we seek in this article to understand the role of predictive mechanisms in the forming and evolving of flocks/swarms by using both numerical simulations and mathematical analyses. This article reviews some basic concepts, important progresses, and significant results in the current studies of collective predictive mechanisms, with emphasis on their virtues concerning consensus improvement and communication cost reduction. Due to these advantages, such predictive mechanisms have great potential to find their way in industrial applications.

\section{Introduction}

ollective behavior coordination in a network of dynamic agents has attracted a lot of attention in recent years, in particular, from physicists, biologists, mathematicians and social scientists. Investigation of the fundamental mechanisms yielding collective behaviors is significant not only for bio-group of animals or group activities of humans (e.g., riots, fashion and escaping panic [1], etc.), but also for nanotechnology applications such as spontaneous magnetization [2]. A large volume of literature has reported some research progress in collectively migrating bacteria [3], insects or birds [4], and in phenomena where groups of organisms or non-living objects reach an ordered or synchronized state such as the one corresponding to fireflies flashing in unison [5] or people clapping in phase during rhythmic applause [6].

The motivation for these studies is to understand the emergence of self-organized collective behaviors in groups 
with inter-agent interactions. Indeed, such systems typically show interesting ordering phenomena as the individuals collectively change their behaviors to a common pattern. Dynamical systems with collective behaviors arise in biological networks at multiple levels of abstraction, from interactions among molecules and cells [7] to the behavioral ecology of animal groups [8]. Flocks of birds, schools of fishes and colonies of bacteria can travel in formation and act as one unit, allowing these creatures to exhibit complex collective behaviors such as formation-keeping during migration, obstacle avoidance, leader selection, and foraging [4].

During the last decades, many biologists, physicists, social experts and interdisciplinary scientists have devoted their work to investigating how flocks/swarms can exhibit high-level globally coordinated collective-behavior based on low-level distributed individual intelligence. To fulfill such a task, the first step may be solving the consensus problem [9], [10], where groups of agents asymptotically agree upon certain quantities of interest like attitude, position, temperature, voltage, etc. Furthermore, exploring distributed computational methods for solving consensus problems has direct applications to real industrial systems such as sensor network data fusion, load balancing, unmanned air vehicles (UAVs), attitude alignment of satellite clusters, congestion control of communication networks, multi-agent formation control, and rendezvous [11]-[13].

Among the previous works on consensus problems, Fiedler [14] and Olfati-Saber and Murray [9] established the theoretical foundations of general consensus problems by investigating the relation between the eigenvalue distribution of the Laplacian matrix $L$ associated with the group topology and some important consensus properties such as the achieved consensus speed and the consensus robustness to time-delays. It was also shown that a network with high algebraic connectivity is robust to both node-failures and edge-failures. To improve the speed of convergence towards consensus for homogeneous networks, Olfati-Saber and Murray proposed a method based on the addition of a few long links to a regular lattice, thus transforming it into a small-world network [15], [16]. On the other hand, for heterogeneous influence networks, Yang et al. [17] showed that, by decreasing the scaling exponent in the associated power law distribution of the influence radius of each node, the ability of the network to reach direction consensus can be significantly enhanced due to the leading roles played by a few hub agents. In addition, if agents can adaptively change their velocities appropriately, the convergence speed can be remarkably improved [18]-[20].

Apart from investigating the consensus mechanisms for biological flocks/swarms, more and more scientists have become interested in the underlying interaction or communication manners. A basic but popular flocking simulation model can be traced back to Reynolds [21], where three elementary rules are prescribed, (i) separation: steer to avoid crowding and collision; (ii) alignment: steer towards the average heading; (iii) cohesion: steer to move towards the average position. These rules have been proven effective and are often used in the design of bio-group dynamic models. In 2003, Gazi and Passino [22] proposed an attractive/repulsive (A/R) swarm model in which the motion of each individual is determined by two factors: (i) attraction to the other individuals at long inter-individual distances; (ii) repulsion from the other individuals at short inter-individual distances. With this model, they proved that the individuals typically form a bounded cohesive swarm in finite time. They later generalized their model into a social foraging swarm model [23] by modifying the attractant/repellent profile, i.e., by additionally considering attraction towards favorable regions (or repulsion from unfavorable regions). Under some suitable circumstances, this improved model guarantees convergence to the favorable regions of the foraging profile. The A/R model of Gazi and Passino has been widely adopted by physicists and biologists to mimic selfdriven particles and biological swarms as it provides conditions for guaranteed cohesion of the swarm [24], [26].

A very popular alignment flock model is the Vicsek model [27], where, at each step, every agent updates its steering direction towards the average direction of its neighbors. With the decreasing external noise or the increasing density of the agents, the collective behavior of the flock undergoes a phase transition from a disordered movement to a coherent collective movement. In 2003, Jadbabaie et al. provided mathematical convergence conditions for the Vicsek model, i.e., all the individuals should be linked at least during some time intervals [28]. In 2007, as illustrated in Figure 1, Couzin designed a Three-Circle model [25], [26] by inserting an orientation area governed by the Vicsek model between the attraction and repulsion areas of the A/R model. The corresponding Three-Circle model yields three typical types of collective behaviors, i.e., swarming, torus-shaped collective motion, and flocking. More precisely, if the internal orientation area is inexistent, the model yields a swarming behavior; if a narrow orientation area exists, a torus-shaped collective motion

Hai-Tao Zhang is with the Department of Control Science and Engineering, Huazhong University of Science and Technology, Wuhan, 430074, P.R. China. Hai-Tao Zhang, Michael ZhiQiang Chen, Guy-Bart Stan, and Jan M. Maciejortsski are with the Department of Engineering, University of Cambridge, Cambridge, CB2 1PZ, U.K. Michael ZhiQiang Chen is also with the Department of Engineering, University of Leicester, Leicester, LE1 7RH, U.K. Tao Zhou (zhutouster@gmail.com) is with both the Department of Modern Physics, University of Science and Technology of China, Hefei 230026, PR China, and the Department of Physics, University of Fribourg, Chemin du Muse, CH-1700 Fribourg, Switzerland. 
will occur; finally, if the orientation area is intensified to a sufficiently large size, the collective behavior will transform from a torus-shaped to a flocking motion. For its wide collective behavior coverage, this novel model has the potential to become one of the most general flocking models in the near future.

Based solely on the currently available information of the network, most of the previous model analyses on flock dynamics have concentrated on properties such as congregation, stabilization, cohesion, and quick consensus. For several decades, biologists have, however, experimentally shown that natural bio-groups possess advanced intelligence, namely predictive intelligence which endows each individual with the capability to predict the future motion of its neighbors according to their past trajectories. As early as in 1959, Woods [29] provided experimental evidences for the existence of certain predictive mechanisms in bee swarm formation. In 1995, Montague et al. [30] proposed simple hebbian learning rules to explain the predictive mechanisms in bees' foraging in uncertain environments. Other researchers focused on the predictive functions of the optical and acoustical apparatuses of bio-groups' individuals, especially the retina and the cortex [31]-[33]. Through extensive bio-eyesight experiments, they found that, when an individual observer prepares to eye-follow the displacement of a visual stimulus, visual adaptation is transferred from the current fixation to the future gaze position. These investigations support the conjecture of the existence of predictive mechanisms inside abundant bio-groups.

Based on these previous experimental results, it seems clear that decisions on the next-step behavior of each individual is not only based on the currently available state information but also on the prediction of future states. This type of behavioral decision based on prediction is used, for example, by a chameleon to capture a fly, by a dog to catch a frisbee, or by a football player to challenge for the point of the first fall. This predictive mechanism reduces the negative influence of information transmission delays within flocks and facilitates the propagation of the group objective or decision information among the individuals of the group. Since nature has chosen to utilize predictive mechanisms, it is reasonable to conjecture that such mechanisms play a very important role in the emergence and evolvement of the abundant biological flocks/swarms. Moreover, development of relevant analysis methods for predictive mechanism can be critical to thoroughly understand and break through the collective performance bottlenecks of industrial multi-agent systems.

For all the reasons mentioned above, one fundamental question that we propose to address in this article is: What exact role is played by the predictive mechanisms embedded in biological flocks/swarms? A physical picture illustrating the prediction-based collective motion paradigm is given in Figure 2 and interpreted as follows: In natural bio-groups, each individual's next-step action is based not only on the current state information (including position, velocity, etc.) of the other (neighboring)

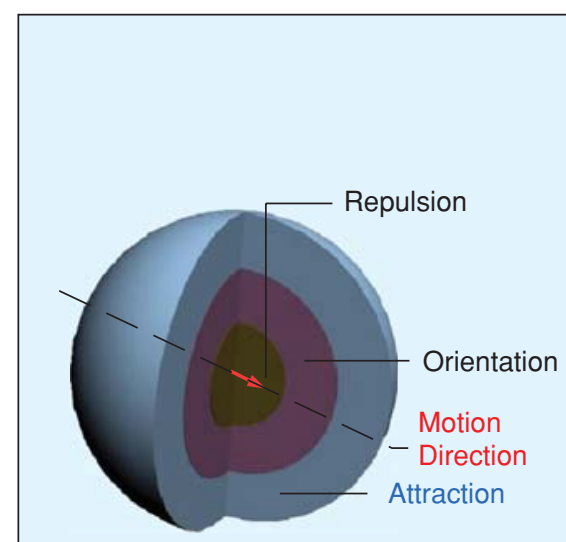

(a)

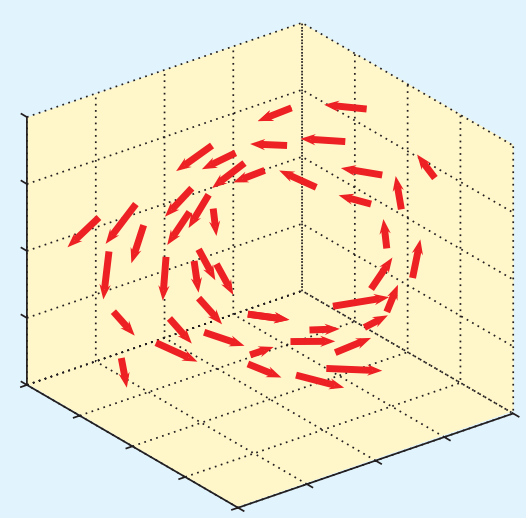

(c)

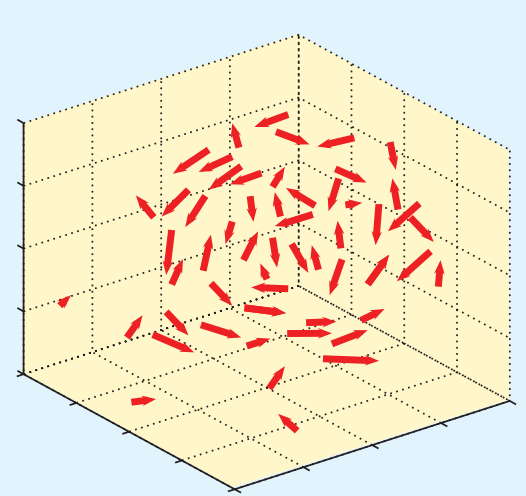

(b)

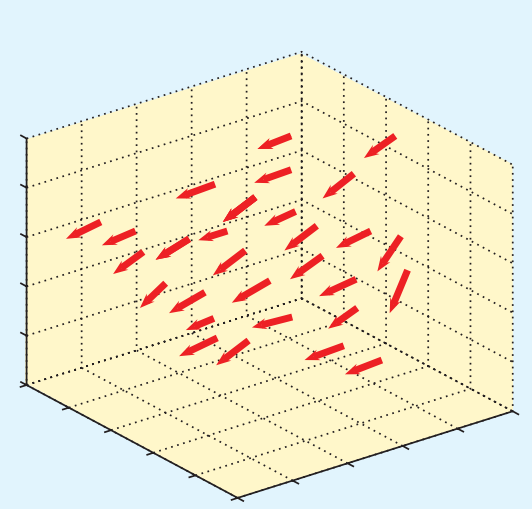

(d)

Figure 1. (Color online) (a) Couzin's Three-Circle model including repulsion, orientation and attraction regions; (b) Swarms: individuals congregating, cohesively repelling and attracting each other, without trying to orient themselves according to their neighbors' direction of motion; (c) Toruses: individuals moving around an empty center; (d) Flocks: individuals moving collectively in one direction. 
agents but also on their predicted future states. More precisely, remembering a few past states of its neighbors and itself, an agent can estimate their future states several steps ahead and then make a decision on its next action to minimize the future state difference with its neighbors.

Meanwhile, collective predictive protocols are beginning to find their way into engineering areas such as autonomous robot formations, sensor networks, and UAVs [11]-[13]. Each agent in these groups typically has limited power to send messages, and thus communication in batch mode, rather than continuous mode, is generally desirable to save energy. This is precisely one of the advantages of predictive protocols. Indeed, as will be shown in this article, predictive protocols typically allow to sharply expand the range of feasible sampling periods and to save costly long range communications.

The objective of this article is to reveal the important role of the predictive mechanisms in the emergence of collective behaviors and to design autonomous and reliable predictive protocols for industrial multi-agent systems. We will present some recently developed theoretical and numerical tools for modeling, analyzing and designing predictive motion protocols for flocks/swarms, both with and without leaders. We will pay special attention to the development of design methodologies that provide industrial multi-agent systems with provably correct cooperative predictive strategies, the characterization of the improvement of the system's collective behavior induced by the predictive capability, and the effective economization of the communication cost owing to such capability.

We will approach the problem in three stages. Firstly, we emphasize the role of predictive mechanisms for some

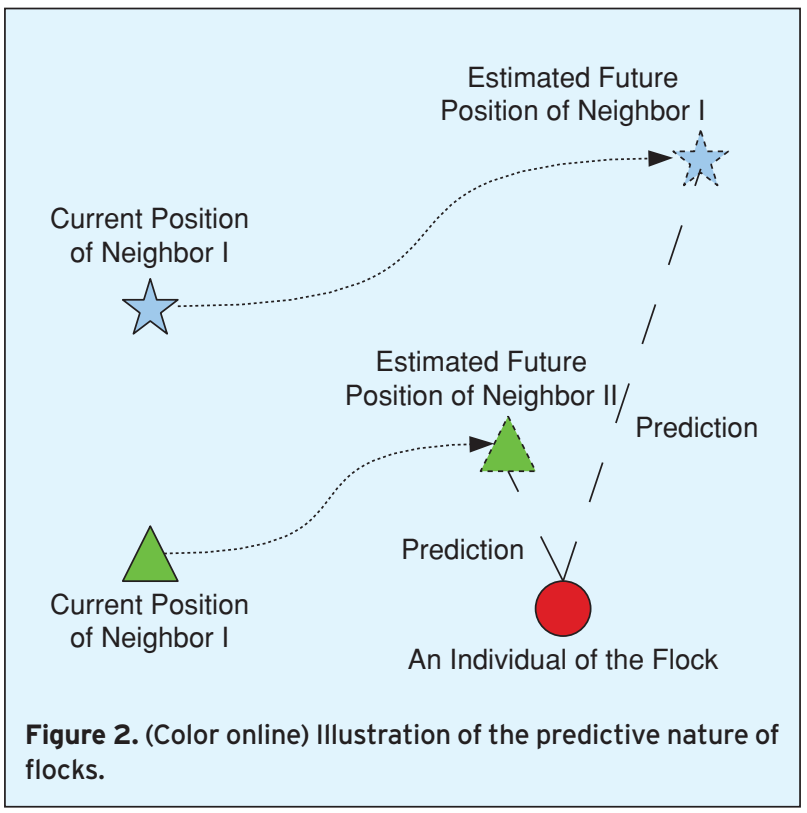

specific types of flocks/swarms with leaders, namely the A/R [22] and Vicsek [27] models. More specifically, a predictive protocol is designed based on the Wattz-Strogatz (WS) small-world connection model [34]. This preliminary study shows that the intelligent predictive capability not only improves the cohesive and formative collective behavior but also reduces the long-range communication cost inside the flock. The power of predictive mechanisms is thus initially demonstrated.

Secondly, in order to evaluate the capability of predictive mechanisms in groups without leaders, we design a predictive protocol for linear dynamic networks. This investigation vividly demonstrates that the predictive protocol can yield faster consensus speeds and remarkably reduce the required sampling frequencies. Moreover, to better understand the collective predictive mechanism, we also give the mathematical analysis to illustrate the improvements endowed by it.

The third focus is the decentralized predictive mechanisms in networks without leaders. In the second stage of our exploration above, we have assumed that the global network information of the flock (state of each individual and topology of the network) is available to each node. In this sense, this method is a centralized or nominal one. Nevertheless, an individual typically lacks global knowledge and, instead, can solely use the observable local information to plan its next movement [4]. Therefore, a decentralized predictive mechanism is more realistic and preferred in practical applications. To develop a decentralized predictive protocol, the question is essential whether the information provided by the local neighbors of an individual is enough to estimate their future states. The proof of this result is established in the third part of the article, after which we design a general decentralized predictive protocol based merely on local observations. As shown through mathematical analyses and numerical simulations, this decentralized protocol exhibits superiority in terms of convergence speed and communication efficiency.

The rest of this article is organized as follows. Section II discusses predictive mechanisms for flocks with leaders, while Section III presents predictive mechanisms for flocks without leaders. Section IV investigates decentralized predictive mechanisms for flocks without leaders. Conclusions are given in Section V.

\section{Predictive Mechanisms for Flocks with Leaders}

To make a rational analysis of the collective predictive mechanisms of flocks with leaders, we consider a typical complex network model, i.e., the WS small-world network model, and use it as the starting framework

4 for the design of such mechanisms. Then, to demonstrate the remarkable improvements endowed by the newly introduced predictive capability, both velocity 
synchronization and position cohesion performances are evaluated for two dominating flocking models, the $\mathrm{A} / \mathrm{R}$ and Vicsek models.

As shown in [34], for flocks with a single leader, it is advantageous to consider a small-world-type network obtained by randomly adding long-range connections from the position of the leader predicted several steps ahead to the current position of a few distant agents called pseudo-leaders. Other non-special agents are called followers. Thus, there are three different kinds of agents: leader (L), pseudo-leaders (P), and followers (F).

\section{A. Predictive Mechanisms}

\section{for Flocks Governed by A/R Model}

In this scenario, the flock is assumed to move in an $m$ dimensional space, the standard A/R function [9], [22]

$$
G\left(d_{p L}\right)=-d_{p L}\left(a-b \cdot \exp \left(-\left\|d_{p L}\right\|_{2}^{2} / c\right)\right)
$$

is added to govern the dynamics of the long-range interaction from the leader (L) to each pseudo-leader (P). $a, b, c$ denote free parameters, $d_{p L}$ is an $m$-dimensional vector pointing from the predicted location of leader $L$ to the current location of a pseudo-leader $p$, and $\left\|d_{p L}\right\|_{2}$ denotes the Euclidian distance between them, here $\|y\|_{2}=\sqrt{y^{T} y}$. For simplicity, in our model, the motion of the leader is known in advance, and will not be affected by any other agent. Additionally, we assume that each pseudo-leader is able to accurately predict the leader's position $H_{p}$ steps ahead.

On the other hand, a weaker $\mathrm{A} / \mathrm{R}$ function, governing the dynamics of the short-range interaction between two arbitrary neighboring agents $i$ and $j$ is given by:

$$
g\left(d_{i j}\right)=-d_{i j}\left(\tilde{a}-\tilde{b} \cdot \exp \left(-\left\|d_{i j}\right\|_{2}^{2} / \tilde{c}\right)\right),
$$

where $d_{i j}$ is an $m$-dimensional vector pointing from the individuals $j$ to $i,\left\|d_{i j}\right\|_{2}=\sqrt{d_{i j}^{T} d_{i j}}$ denotes the Euclidian distance between them, and the parameters $\tilde{a}, \tilde{b}$ and $\tilde{c}$ are typically much smaller than $a, b$, and $c$, respectively. We denote by $r$ the radius of the influence circle of each agent (see Figure 3). Any two agents of the type F-F, P-P and L-F which are within a distance $r$ of each other are connected by a link whose strength is characterized by the $\mathrm{A} / \mathrm{R}$ function in Eq. (2). Conversely, irrespective of their relative distance, L-P agents are always connected by a link whose strength is described by the A/R function in Eq. (1). Note that the leader is never influenced by other agents. To decrease the prediction cost, prediction is only allowed for the L-P interactions. Bearing in mind the physical meaning of $\mathrm{A} / \mathrm{R}$ functions [9], the 5 positions of a pseudo-leader $x_{p}$ and a follower $x_{i}$ is determined by

$$
\begin{aligned}
x_{p}(k+1)= & x_{p}(k)+\underbrace{G\left(d_{p L}\left(k+H_{p}\right)\right)}_{\text {long link to the leader }} \\
& +\underbrace{\sum_{j \neq L, d_{p j}(k) \leq r} g\left(d_{p j}(k)\right),}_{\text {neighboring links }}
\end{aligned}
$$

and

$$
x_{i}(k+1)=x_{i}(k)+\underbrace{\sum_{j, d_{i j}(k) \leq r} g\left(d_{i j}(k)\right)}_{\text {neighboring links }},
$$

respectively.

In these equations, $k$ denotes the current discrete-time instant, and $d_{p L}\left(k+H_{p}\right)$ represents the $m$-dimensional vector pointing from the leader's position $H_{p}$ steps ahead to the current position of a pseudo-leader. In this way, unlike the routine flocking strategies proposed in [21]-[23], [35], a small-world interaction dynamics incorporating a specific predictive mechanism is considered. The discrete-time dynamical system (3), (4) is associated with an interaction network, where each agent is considered as a node, and two agents are connected either if their relative distance is smaller than a predefined radius of influence or if there exists a direct long-range connection between the considered agent and the leader. Clearly, the structure of this interaction network changes in time since the position of each agent is varying over time. It, however, always displays the small-world property due to the existence of permanent long-range links between the pseudo-leaders and the leader.

How can this predictive protocol, based on a few longrange interactions, improve the flocking performance? The answer lies in the way information flows in the network as illustrated in Figure 4 [37]. The farthest agent $i_{1}$ directly communicating with agent $i$ is among the ones at the rim of the influence circle centered on agent $i$. Analogously, the farthest agent $i_{2}$ directly influenced by $i_{1}$ is also located at the rim of the circle centered on agent $i_{1}$, and so forth. Finally,

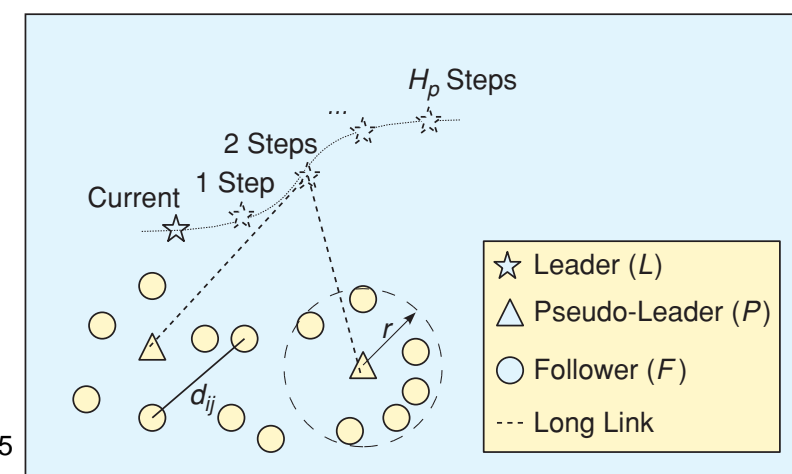

Figure 3. Predictive mechanism in small-world networks. 
the information of agent $i$ reaches agent $j$ in $H_{p}$ discrete-time steps. Accordingly, if agent $i$ acts as a pseudo-leader who can accurately predict the behavior of the leader $H_{p}$ steps ahead, at time step $k$, agent $j$ 's motion is affected by the exact current position of the leader $x_{L}(k)$. Thereby, agent $j$ can adhere to the leader more tightly, the flock's formation is more likely to be kept stable, and the coherence of the whole flock is thus improved effectively. Note that the predictive mechanism is valid only if the leader's motion is accurately predictable (e.g., following a known continuous trajectory without large fluctuation or some erratic movement). Fortunately, in the real biological world, the flock leader typically moves in some predictable pattern.

Now two important questions arise: How many pseudo-leaders are required to yield a satisfactory group behavior? And how many steps should be predicted by each pseudo-leader? In order to extract the role of the prediction horizon $H_{p}$ and the number of pseudo-leaders $N_{p l}$, we study their influences on the velocity synchronization and position cohesion performance indexes $J_{v}$ and $J_{p}$ which are respectively defined as:

$$
\begin{aligned}
& J_{v}=\frac{1}{N-1} \sum_{i=1, i \neq L}^{N}\left\|v_{i}-v_{L}\right\|_{2}, \\
& J_{p}=\frac{1}{N-1} \sum_{i=1, i \neq L}^{N}\left\|d_{i L}\right\|_{2} .
\end{aligned}
$$

$J_{v}$ measures the velocity synchronization or formation performance of the flock, where $v_{L}$ and $v_{i}$ denote the velocity vectors of the leader and the $i$ th agent ( $\mathrm{F}$ or P); $J_{p}$ measures the cohesion performance of the flock, with $d_{i L}$ denoting the distance between agent $i$ (F or P) and the leader.

In Figure 5(a), we consider $H_{p}$ as a fixed parameter and display the curves of $J_{v}$ with increasing $N_{p l}$, while Figure 5(b), on the contrary, displays the curves of $J_{v}$ with increasing $H_{p}$ and fixed $N_{p l}$. It can be seen from Figure 5(a) that the curves decrease sharply at the

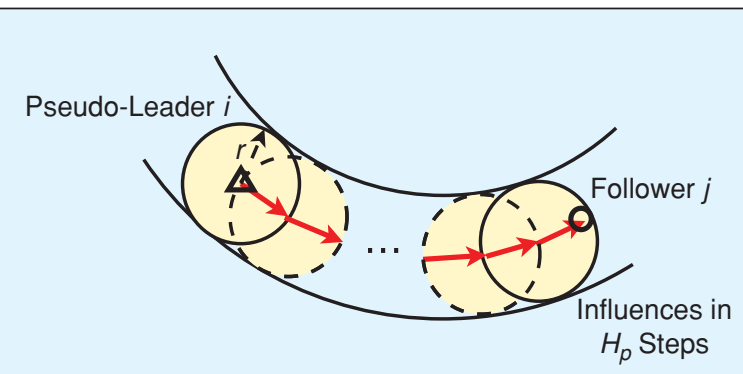

Figure 4. (Color online) Information flow within a network endowed with a small-world predictive mechanism. If the pseudo-leader $i$ is able to accurately predict the state of the leader $H_{p}$ steps ahead, then the follower $j$, which is influenced by $i$ after $H_{p}$ steps, will be affected by the current location of the leader. beginning and reach a minimum $N_{p l}^{*}$ before increasing more slowly. The presence of a minimum at low values of $N_{p l}$ implies that adding just a few pseudo-leaders to the system, which transforms the flock topology from a strongly localized network into a small-world one, will improve the flocking performance optimally in terms of $N_{p l}$. If more pseudo-leaders than the optimal value $N_{p l}^{*}$ are added, the flock formation performance starts to worsen as these extra pseudo-leaders become redundant. On the other hand, we also see that increasing $H_{p}$ can help in reducing $J_{v}$ in two ways: (i) it decreases $J_{v}$ for a fixed value of $N_{p l}$; (ii) it reduces the optimal value $N_{p l}^{*}$. Compared with Figure 5(a), the $J_{v}$ curves in Figure 5(b) also possess a clear minimum. The presence of this minimum implies that the flock formation performance can be remarkably improved with proper predictive capability. Nevertheless, too much vision into the future, namely over-prediction, will worsen the flocking performance as measured by $J_{v}$.

Next, we investigate the effects of $H_{p}$ and $N_{p l}$ on the other important flock performance index $J_{p}$. It can be seen from Figure 5(c) that the curves fall sharply at the beginning and asymptotically approach a stable value as $N_{p l} / N \rightarrow 1$. Contrary to Figure 5(a), no clear minimum exists in Figure 5(c). As illustrated in Figure 5(c), increasing $H_{p}$ yields smaller values of $J_{p}$. Compared with Figure 5(c), the curves of Figure 5(d) do possess a minimum, which is reached at a fairly large $H_{p}$. As a consequence, the compactness of the flock is clearly dependent on $H_{p}$ and over-prediction can be detrimental to it. Furthermore, increasing the number of pseudo-leaders can improve the cohesive flocking performance. Actually, when $N_{p l}$ exceeds a certain value, $J_{p}$ decreases so slowly that almost no benefit can be gained by further increasing $N_{p l}$. In brief, suitable insight into the future and moderate number of pseudo-leaders are preferred.

The influence of pseudo-leaders extracted here can easily find examples in nature. As shown in Figure 6, a few informed individuals (or pseudo-leaders) of a fish school or a bee swarm can guide the whole group towards the target (or help the group follow the trajectory of the leader) [24], [25], [36]. The role of pseudo-leaders has also been investigated by Couzin [35], who showed that the larger the group the smaller the proportion of informed individuals needed to guide the group, and that only a very small proportion of informed individuals is required to achieve remarkable accuracy. Interestingly, compared with Couzin's results, we have shown through the numerical simulations presented in Figure 5 that the incorporation of predictive intelligence can even further 6 reduce the required number of pseudo-leaders.

More significantly, as shown in Figure 5, to achieve a fixed flocking performance, larger predictive capability 
(larger values of $H_{p}$ ) can compensate for the insufficiency of the pseudo-leaders $\left(N_{p l}\right)$ and vice versa. This rule is useful for industrial applications since one can optimize the performance of the dynamic flock by evaluating the costs of the increased predictive capability and the addition of the long-range connections.

\section{B. Predictive Mechanisms for Flocks Governed by the Vicsek Model}

The role of predictive mechanisms highlighted in Subsection II-A is not merely confined to A/R flocks but quite general. To verify this, we now incorporate the predictive mechanism proposed for small-world interaction patterns in the previous section into another well-accepted flocking model, i.e., the Vicsek model [27] and compare the synchronization performance of the predictive small-world Vicsek model with the one of a classical Vicsek model.

In this model, the velocities $v_{i}$ of the $N$ agents composing the group are determined simultaneously at each discrete-time instant, and the position of the $i$ th agent is updated according to

$$
x_{i}(k+1)=x_{i}(k)+v_{i}(k),
$$

where $v_{i}(k)$ denotes the velocity vector of agent $i$ at time $k$. For each agent the velocity vector, $v_{i}(k)$, is characterized by a constant magnitude $v$ and by a direction $\theta_{i}(k)$ whose dynamics is given by

$$
\theta_{i}(k+1)=\left\langle\theta_{i}(k)\right\rangle_{r}+\Delta \theta_{i}
$$

where $\left\langle\theta_{i}(k)\right\rangle_{r}$ denotes the average direction of all the agents' velocity vectors within a circle of radius $r$ centered on agent $i$, i.e.,

$$
\left\langle\theta_{i}(k)\right\rangle_{r}=\left\{\begin{array}{l}
\arctan \left[\left\langle\sin \left(\theta_{i}(k)\right)\right\rangle_{r} /\left\langle\cos \left(\theta_{i}(k)\right)\right\rangle_{r}\right] \\
\text { if }\left\langle\cos \left(\theta_{i}(k)\right)\right\rangle_{r} \geq 0 \\
\arctan \left[\left\langle\sin \left(\theta_{i}(k)\right)\right\rangle_{r} /\left\langle\cos \left(\theta_{i}(k)\right)\right\rangle_{r}\right]+\pi \\
\text { otherwise, }
\end{array}\right.
$$

where $\left\langle\sin \left(\theta_{i}(k)\right)\right\rangle_{r}$ and $\left\langle\cos \left(\theta_{i}(k)\right)\right\rangle_{r}$ denote the average sine and cosine values, and $\Delta \theta_{i}$ represents a random ${ }^{7}$ noise obeying a uniform distribution in the interval $[-\eta / 2, \eta / 2]$.
As shown in Figure 7(a) the particles are distributed in a square of dimension $[0, L] \times[0, L]$, and the trajectory of the leader is a circle centered at $(L / 2, L / 2)$ with radius $R=L / 6$ so that the direction of the leader changes constantly. The small-world predictive connection framework shown in Figure 3 is used together with the Vicsek model. Hence, there are always $N_{p l}$ individuals having long-range predictive interactions with the predicted motion of the leader $H_{p}$ steps ahead. It is shown in Figure 7(b) that drastic improvement of the velocity synchronization performance can be achieved with moderate prediction horizons. Similar to the case of the A/R model shown in Subsection II-A, one can also conclude that suitable insight into the future and moderate number of pseudo-leaders is preferable.

To further verify the generality of these conclusions, we have also applied the proposed predictive mechanism to one of the most general flocking modelsCouzin's three-circle model [25], [26]. The corresponding results also strongly suggest that prediction is beneficial to the flock performance. In summary, by designing a predictive protocol within a small-world connection 
framework, we have hereby given some new convincing evidence for the existence of predictive intelligence in bio-groups with leaders, and shed some light on the important role of such predictive mechanisms for the collective behavior coordination in natural flocks/swarms.

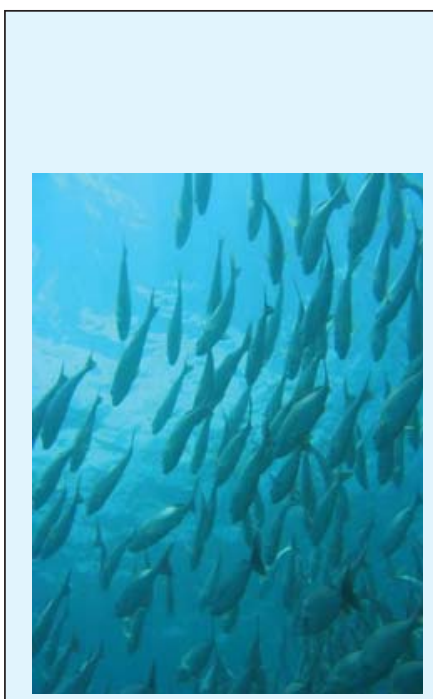

(a)

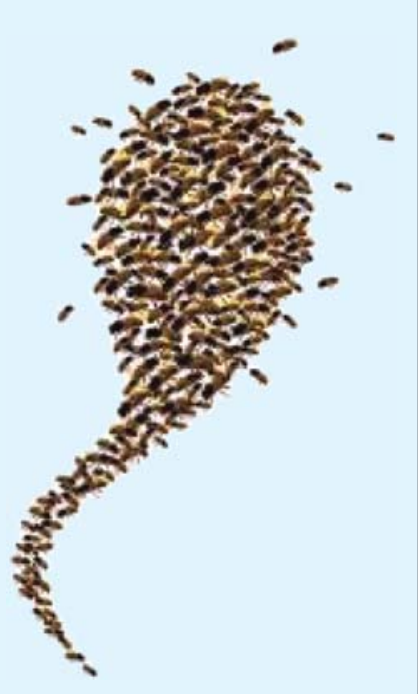

(b)
Figure 6. (Color online) Two examples of pseudo-leaders found in nature, (a) A few informed individuals within a fish school are known to be able to influence the ability of the school to navigate towards a target; (b) Within a honeybee swarm, only about $5 \%$ of the bees play a pseudo-leader role, which helps in guiding the group to a new nest site [36].

\section{Centralized Predictive Mechanisms for Flocks Without Leaders}

It is well-accepted by biologists that natural bio-groups do not necessarily have leaders [38]. In the following two sections we design a predictive protocol for consensus networks without leaders. In particular, to reveal the role of predictive mechanisms in consensus problems, we focus our discussion on consensus optimization using model predictive control (MPC). Unlike the numerical simulation approach used in the previous section, we will mainly use mathematical analysis to carry out our work.

\section{A. Preliminary Concepts}

We represent a network of interacting agents by a digraph $G=(\mathcal{V}, \mathcal{E}, A)$, where $\mathcal{V}=\left\{v_{1}, \ldots, v_{N}\right\}$ is the set of nodes representing the agents, $\mathcal{E} \subset \mathcal{V} \times \mathcal{V}$ is the set of edges, and $A$ is the $N \times N$ adjacency matrix with $a_{i j} \geq 0$ denoting the edge weight from node $i$ to node $j$. No self-cycle is allowed, hence $a_{i i}=0$ for all $i$. The Laplacian matrix $L$ is defined as $l_{i i}=\sum_{l \neq i}^{N} a_{i l}$ and $l_{i j}=-a_{i j}, \forall i \neq j$. We denote by $d_{\max }=\max _{i}\left(l_{i i}\right)$ the maximum out-degree of $G$. A digraph $G$ is called balanced if $\forall i, \sum_{j} a_{i j}=\sum_{j} a_{j i}$. If any two distinct nodes can be connected via a path that follows the direction of the edges of the digraph, this network is called strongly connected. $x_{i}(t) \in \mathbb{R}$ denotes the state of node $i$, which could represent a certain physical quantity such as attitude, position, temperature, voltage, and so on. Generally, a network is said to have reached

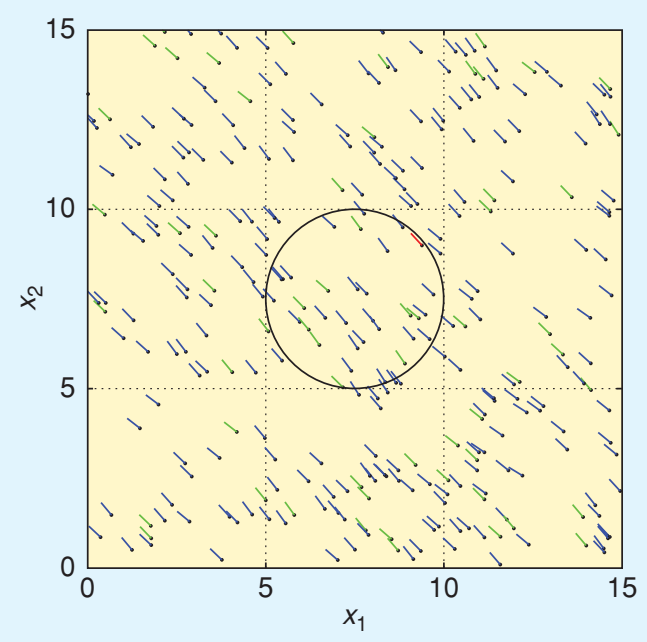

(a)

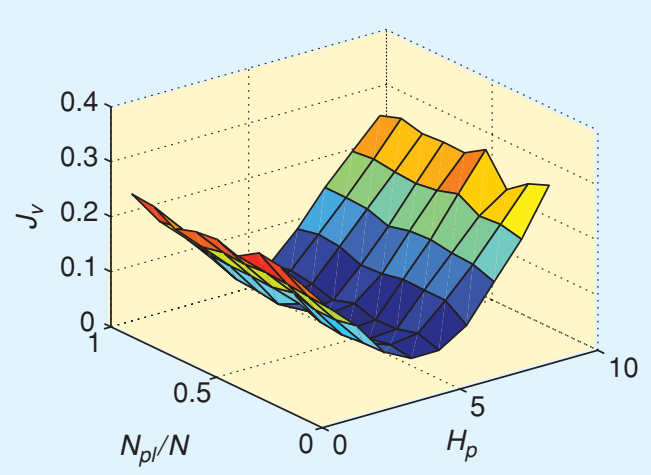

(b)

Figure 7. (Color online) (a) Snapshot of the predictive Vicsek flock at the 12th running step. The red particle denotes the leader; the green particles represent the pseudo-leaders and the blue particles denote followers. The centered black circle outlines the trajectory of the leader. For these simulations, the prediction herizon is $H_{p}=4$. (b) Velocity synchronization index $J_{v}$ as a function of the parameters $H_{p}$ and $N_{p}$. The parameters of this simulation have been chosen to be $L=15, \eta=0.1, v=0.15, N=300$, $R=L / 6$ and each point is an average over 100 independent runs. 
consensus (or agreement) if and only if $x_{i}=x_{j}$ for all $i, j \in \mathcal{V}$. Whenever the nodes of a network are all in agreement, their common value is called the group decision value. If this value is $\bar{x}(0)=\left(\sum_{i=1}^{N} x_{i}(0)\right) / N$, the network is said to have reached the average-consensus.

\section{B. Consensus Model Predictive Control for Agents with Integrator Dynamics}

We consider the following discrete-time model for the dynamics of agent $i$ :

$$
x_{i}(k+1)=x_{i}(k)+\epsilon u_{i}(k), \quad i=1, \ldots, N
$$

where $\epsilon$ and $u_{i}(k)$ denote the sampling period and the control input, respectively. For agents with such linear dynamics, average-consensus is typically asymptotically reached using the routine consensus protocol

$$
u_{i}(k)=\sum_{j=1}^{N} a_{i j} \Delta x_{i, j}(k), \quad i=1, \ldots, N,
$$

where $\Delta x_{i, j}(k)=x_{j}(k)-x_{i}(k)$ denotes the difference between the state of the $i$ th and the $j$ th agents [9]. Using the routine protocol, the corresponding network dynamics is given by

$$
x(k+1)=P_{\epsilon} x(k)
$$

with $P_{\epsilon}=I_{N}-\epsilon L$. If $G$ is strongly connected and balanced, and the sampling period $\epsilon \in\left(0,1 / d_{\max }\right)$, then the routine consensus protocol (8) ensures global asymptotic convergence to consensus [9].

To integrate a predictive mechanism into the routine protocol, we introduce an additional prediction term $v_{i}(k)$ into Eq. (8):

$$
u_{i}(k)=\sum_{j=1}^{N} a_{i j} \Delta x_{i, j}(k)+v_{i}(k) .
$$

Accordingly, the network dynamics becomes

$$
x(k+1)=P_{\epsilon} x(k)+v(k)
$$

with $v(k)=\left[v_{1}(k), \ldots, v_{N}(k)\right]^{T}$. Using the above dynamics, one can predict the future states of the network $H_{p}$ steps ahead as follows:

$$
X(k+1)=P_{X} x(k)+P_{U} U(k)
$$

with

$$
\begin{aligned}
X^{T}(k+1) & =\left[x^{T}(k+1), \ldots, x^{T}\left(k+H_{p}\right)\right], \\
U^{T}(k) & =\left[v^{T}(k), \ldots, v^{T}\left(k+H_{p}-1\right)\right], \\
P_{X} & =\left[P_{\epsilon}^{T}, \ldots,\left(P_{\epsilon}^{H_{p}}\right)^{T}\right]^{T}
\end{aligned}
$$

and $P_{U}$ given in Appendix A.
Accordingly, the future evolution of the network's state difference can be predicted as follows:

$$
\begin{aligned}
\Delta X(k+1) & =\left[\Delta x(k+1)^{T}, \ldots, \Delta x\left(k+H_{p}\right)^{T}\right]^{T} \\
& =P_{X E} x(k)+P_{U E} U(k)
\end{aligned}
$$

with $P_{X E}=E P_{X}, P_{U E}=E P_{U}, E=\operatorname{diag}(e, \ldots, e), e=$ $\left[e_{1,2}^{T}, \ldots, e_{1, N}^{T}, e_{2,3}^{T}, \ldots, e_{2, N}^{T}, \ldots, e_{N-1, N}^{T}\right]^{T}, e_{i, j}=e_{i}-e_{j}$, $e_{j}=\left[0, \ldots, 0,1^{j t h}, 0, \ldots, 0\right]$, the state difference vector

$$
\begin{aligned}
\Delta x(k+m)= & {\left[\Delta x_{1,2}^{T}(k+m), \ldots, \Delta x_{1, N}^{T}(k+m),\right.} \\
& \Delta x_{2,3}^{T}(k+m), \ldots, \Delta x_{2, N}^{T}(k+m), \ldots, \\
& \left.\Delta x_{N-1, N}^{T}(k+m)\right]^{T},
\end{aligned}
$$

and $\Delta x_{i, j}(k+m)=x_{i}(k+m)-x_{j}(k+m)=e_{i, j} x(k+m)$.

In this setting, the problem is thus to design the additional term $v_{i}(k)$ in Eq. (10) in order to optimally (i.e., as quickly as possible) reduce future disagreements among individuals of the group. To solve this optimal consensus problem, we first define the cost function as below:

$$
J(k)=\|\Delta X(k+1)\|_{Q}^{2}+\|U(k)\|_{R}^{2},
$$

where $Q$ and $R$ are compatible weighting matrices which are generally set as $Q=q I_{H_{p} \times N(N-1) / 2}(q>0)$ and $R=I_{H_{p} \times N}$, and $\|M\|_{Q}^{2}=M^{T} Q M$. In Eq. (14), the first term penalizes the disagreement in the network over the future $H_{p}$ steps, while the second term penalizes the additional MPC energy $v(k)$. To minimize the cost function $J(k)$, we compute $\partial J(k) / \partial U(k)=0$, which is a linear function of $x(k)$ and $U(k)$, and consequently obtain the optimal MPC action $v(k)=P_{\mathrm{MPC}} x(k)$ with

$P_{\mathrm{MPC}}=-\left[I_{N}, \mathbf{0}_{N}, \ldots, \mathbf{0}_{N}\right]\left(P_{U E}^{T} Q P_{U E}+R\right)^{-1} P_{U E}^{T} Q P_{X E}$.

A schema illustrating the proposed consensus MPC protocol is shown in Figure 8.

In particular, the global topology and state of the network is utilized to predict the future state difference of the network and to compute the future $H_{p}$ control inputs which are optimal with respect to the predicted cost function (14). At the next discrete-time instant, only the first control input is implemented, after which a new network state measurement is taken and the whole process is started again.

This new consensus algorithm is characterized by four important properties:

9 1) For symmetric (i.e., $a_{i j}=a_{j i}$ ) and strongly connected networks, the state matrix $P_{\epsilon}+P_{\mathrm{MPC}}$ is also symmetric and has the same eigenvectors as $P_{\epsilon}$; 
2) For strongly connected and balanced networks, the matrix $P_{\mathrm{MPC}}$ is also balanced in the sense that $P_{\mathrm{MPC}} \mathbf{1}=P_{\mathrm{MPC}}^{T} \mathbf{1}=\mathbf{0}$ with $\mathbf{1}=[1, \ldots, 1]_{N \times 1}^{T}$ and $\mathbf{0}=0 \cdot \mathbf{1}$

3) For strongly connected and balanced networks, average-consensus will be achieved if and only if the matrix $P_{\epsilon}+P_{\mathrm{MPC}}$ has a simple eigenvalue at 1 and all its other eigenvalues inside the open unit circle;

4) For strongly connected and balanced networks, if we denote each entry of $P_{\epsilon}+P_{\text {MPC }}$ by $\beta_{i j}(i, j=1, \ldots, N)$, then average-consensus will be achieved provided that $\beta_{i j} \geq 0(i \neq j)$ and $\beta_{i i} \in(0,1]$.

The mathematical proofs of these properties, beyond the scope of this article, are provided in an extended paper [43].

This proposed MPC protocol yields the following two improvements compared with the routine protocol:

C1: The feasible range of the sampling period $\epsilon$ is remarkably expanded;

C2: For feasible values of $\epsilon$, the convergence speed is significantly increased.

To illustrate the advantages $\boldsymbol{C 1}$ and $\boldsymbol{C 2}$, we present some numerical results comparing the convergence speeds of the MPC and routine protocols for an all-to-all network of 10 nodes. Since the objective is to reach average-consensus, the instantaneous disagreement index is typically set as $D(k)=\|x(k)-\mathbf{1} \bar{x}(0)\|_{2}^{2} \quad$ (here $\left.\|x\|_{2}=\left(x^{T} x\right)^{1 / 2}\right)$ and the consensus steps $T_{c}\left(D_{c}\right)$ is defined as the number of steps required for $D(k)$ to reach a specific small threshold value, $D_{c}$. Based on these definitions, $1 / T_{c}\left(D_{c}\right)$ gives a reasonable measurement of the consensus speed. As shown in Figure 9(a), the addition of the predictive control term defined in (15) yields a drastic increase in convergence speed towards consensus. In particular, for $\epsilon<1 / d_{\max }=1 / 9\left(d_{\max }=\max _{i}\left(l_{i i}\right)\right)$, the convergence speed is increased more than 20 times

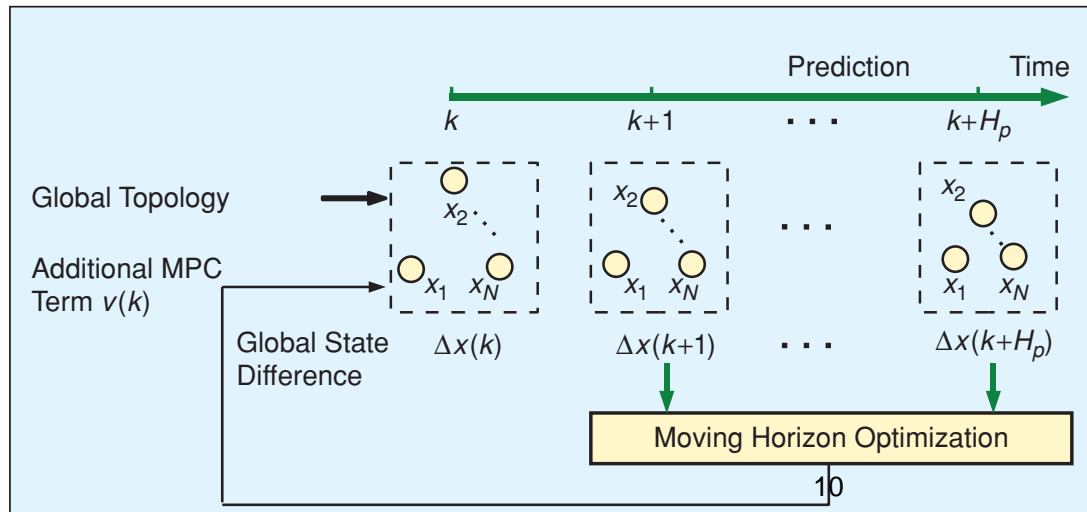

Figure 8. Nominal MPC protocol. (measured by the slope of the $D(k)$ curve) by using the proposed MPC protocol. Furthermore, even when the routine convergence condition is violated, i.e., $\epsilon>1 / d_{\max }$, it is observed that the MPC protocol still allows asymptotic convergence with high speed.

To study the effects of the predictive control mechanism on the feasible convergence range of $\epsilon$, we examine in Figure 9(b) the average consensus steps $T_{C}(0.01)$ of these two strategies with different $\epsilon$ 's. In this plot, for each value of $\epsilon, T_{c}$ denotes the average-consensus steps over all the successful convergence runs. Compared with the routine protocol, the MPC protocol allows for a significant increase in the consensus speed $T_{c}^{-1}$ (by a factor between 6 and 20 in our simulations), which further illustrates the improvement $\boldsymbol{C} 2$.

At this stage, one may ask how this kind of predictive control protocol can improve the consensus performance so remarkably. This can be explained in terms of the distribution of the state matrix eigenvalues as illustrated in Figure 10. Indeed, since the eigenvalue cluster of $P_{\epsilon}+P_{\mathrm{MPC}}$ is typically much smaller and closer to the origin of the complex plane than the one of $P_{\epsilon}$, the predictive control protocol generally exhibits faster consensus. When $\epsilon<1 / d_{\max }$ [see Figure 14(a)], the two eigenvalue clusters are both kept inside the asymptotic stability region, i.e., the unit circle in the complex plane. On the contrary, when $\epsilon>1 / d_{\max }$ (see Figure 14(b)), some of the eigenvalues of $P_{\epsilon}$ start escaping the unit circle, making the disagreement function diverge, whereas all the eigenvalues of $P_{\epsilon}+P_{\mathrm{MPC}}$ remain inside the unit circle, which guarantees its asymptotic convergence.

Furthermore, to verify the generality of these conclusions, we have also applied the proposed MPC protocol to partially linked networks. The corresponding results for partially linked networks are qualitatively similar (C1 and $\boldsymbol{C} 2$ also hold in this case), except that the performance improvements are slightly reduced due to the increased information flow constraints imposed by the network topology.

In summary, based on the accurate predictive capability embedded in each node, we have designed a predictive control protocol for flocks without leader. We presented analytical results as well as numerical simulations to demonstrate its main advantages, namely:

1) Just a little vision into the future leads to significant increases in convergence speeds;

2) The feasible sampling period range can be sharply 


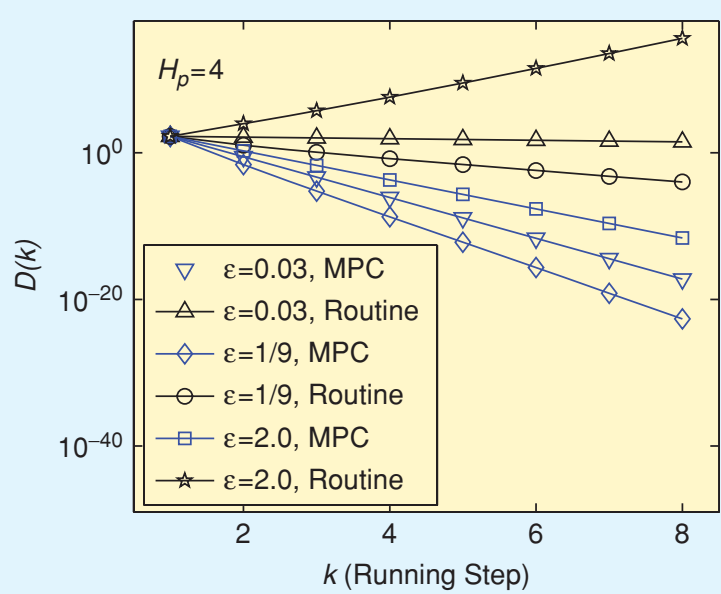

(a)

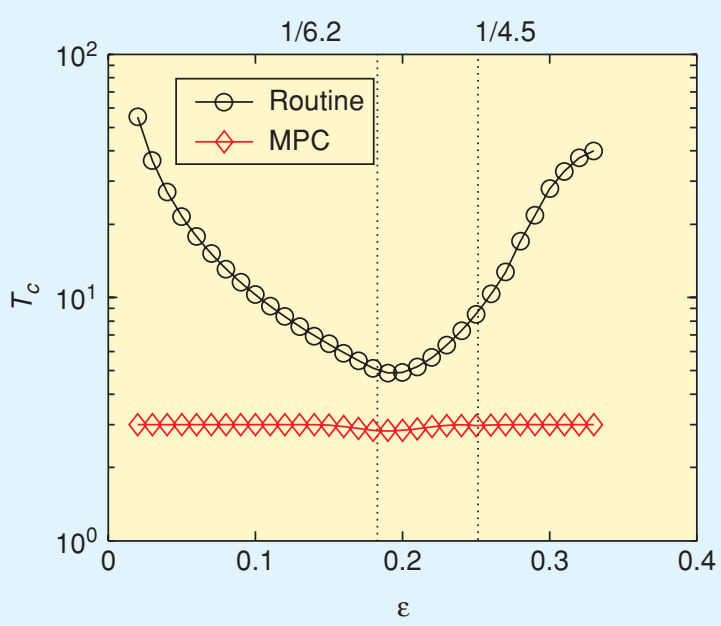

(b)

Figure 9. (Color online) (a) Time-evolution of the instantaneous disagreement $D(k)$ of the routine (black) and MPC (blue) protocols on an all-to-all, symmetric, strongly connected network of $N=10$ nodes; (b) Average-consensus steps $\left(T_{c}(0.01)\right)$. For these simulations, 500 independent runs are considered for each value of $\epsilon_{1} H_{p}=4, q=2, D_{c}=0.01$, entries $I_{i j}(i \neq j)$ of $L$ are selected randomly in $[-1,0)$ such that the resulting network is strongly connected and balanced, and $x_{i}(0)(i=1, \ldots, N)$ is selected randomly in $[0,15]$. The associated values of $d_{\max }$ lie in $[4.5,6.2]$. The vertical dotted lines correspond to the minimum and maximum values of $1 / d_{\max }$.

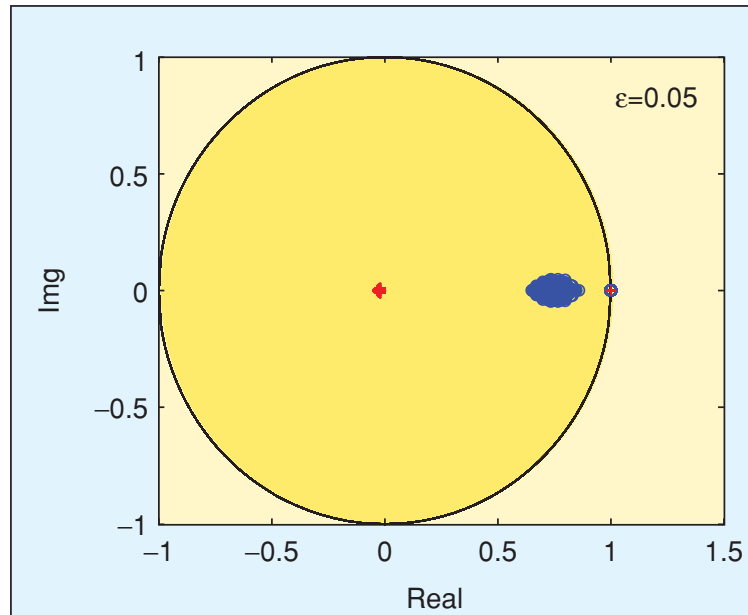

(a)

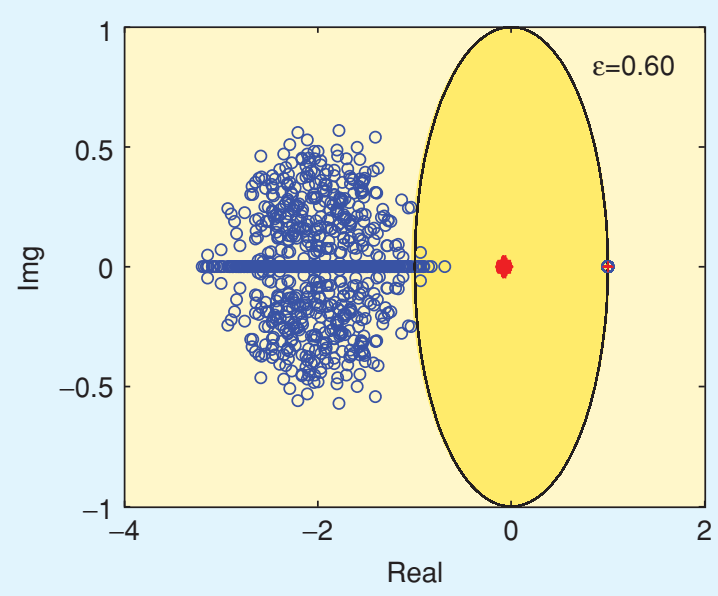

(b)

Figure 10. (Color online) Eigenvalue distributions for different $\epsilon$. Blue $(\bigcirc)$ and red $(+)$ represent the eigenvalues of $P_{\epsilon}$ and $P_{\epsilon}+P_{\mathrm{MPC}}$ over 100 runs, respectively. The black circle denotes the unit circle in the complex plane. Here, $H_{p}=4, q=2$, and each entry $I_{i j}(j \neq i)$ of $L$ is chosen randomly in $[-1,0)$ such that the resulting network is strongly connected and balanced. The associat ed values of $d_{\max }$ lie in $[4.5,6.2]$.

expanded using this predictive mechanism, giving the MPC consensus protocol the potential to effectively save communication energy.

In other words, by introducing some predictive mechanisms, it is possible either to significantly enhance the consensus speed obtainable under the constraint of a fixed communication energy or to decrease the communication energy required to ensure a prescribed consensus speed.

For natural science, this observation plays an important role as it implies that predictive mechanisms may extensively exist in abundant natural bio-groups without leaders. Moreover, it helps explain why individuals of biological swarms, such as fireflies and deep-sea fish, do not communicate very frequently all along but just now and then during the whole dynamic process. This may be explained by the fact that predictive intelligence embedded in each individual can compensate for the need of fre${ }^{11}$ quent communication, which coincides with the flock's batch communication phenomena observed recently [35], [39]. 


\section{Decentralized Predictive Mechanism for Flocks Without Leaders}

Generally, collective behaviours emerge despite the fact that each individual lacks global knowledge of the network state and network topology. Typically, individuals solely have access to some local information provided by their neighbors to plan their motion [4]. In Section III, however, it has been assumed that the global state and topology of the flock is available to every individual of the group. In this sense, the MPC protocol proposed in Section III is a nominal or centralized control one. In this section, we instead design a decentralized predictive protocol, in which each individual can merely estimate a "local" dynamic model of interaction by using the neighboring information it observes and thereby make predictions about its neighbors' future states.

\section{A. Prediction Based on Local Information}

To design a decentralized MPC protocol, an accurate local model, derived and iterated independently by each individual, is required. Thus, the first question is determining whether sole knowledge of local neighbors information by each individual is enough to obtain an accurate dynamical

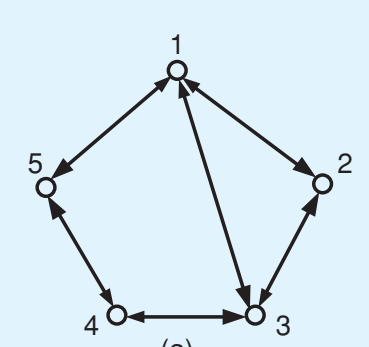

(a)

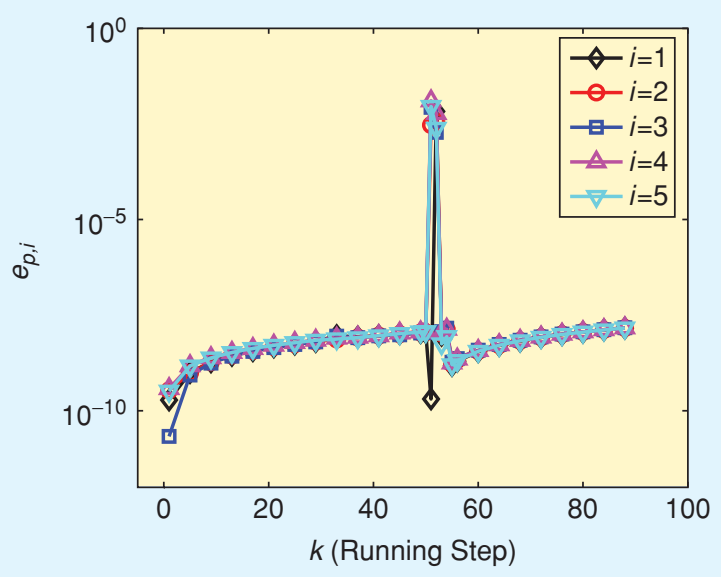

(c)

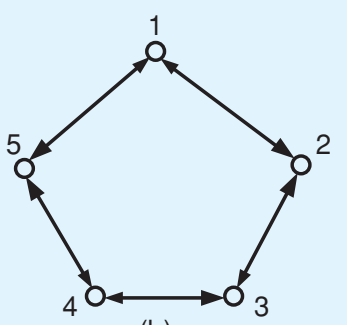

(b)
Figure 11. (Color online) The network topology is switched from (a) to (b) at the 47th running step; (c) gives the instantaneous prediction error $e_{p, i}(k)$. Here, $\epsilon=0.35, a_{i j}=1$ if $(i, j) \in \mathcal{E}$, and $a_{i j}=0$ otherwise, the initial state of each node is selected randomly in the range $[0,150]$, and each point is an average over 100 independent runs. local model. To be shown next, the answer is affirmative provided that the individuals keep record of sufficiently long past state sequences of themselves and their neighbors.

Similar to Section III, we consider the linear discretetime network model (9), based on which the $m$-step-ahead future state of an individual $i$ can easily be derived:

$$
x_{i}(k+m)=e_{i} P_{\epsilon}^{m} x(k),
$$

where $e_{i}=\left[0, \cdots, 0,1^{i t h}, 0, \cdots, 0\right]$. It seems that the global information of the network including the state matrix $P_{\epsilon}$ and the current state $x(k)$ are required to predict the future states of an arbitrary individual of the network. In the following paragraphs, however, we show that, even if such global information is unavailable to each node of the network (which is typically the case in natural bio-groups and engineering multi-agent systems), each individual may still be capable of estimating its own and its neighbors' future states by using the present and past information it collects from its neighbors.

More precisely, let $Z_{i}(k)=\left[z_{1, i}^{T}(k), \cdots, z_{N, i}^{T}(k)\right]^{T}$ denote the historical state sequence of length $N$ for the $i$ th individual with $z_{l, i}(k) \triangleq x_{i}(k+1-l), l=1, \cdots, N$. Using (9), it is easy to show that the following relations exist

$$
x(k-N)=\Phi_{i}^{-1} Z_{i}(k),
$$

and

$$
x_{j}(k+1)=B_{j, i} Z_{i}(k), \quad j=1, \ldots, N
$$

with

$$
\Phi_{i} \triangleq\left[\left(e_{i} P_{\epsilon}^{N}\right)^{T}, \ldots,\left(e_{i} P_{\epsilon}\right)^{T}\right]^{T}
$$

and

$$
B_{j, i} \triangleq e_{j} P_{\epsilon}^{N+1} \Phi_{i}^{-1},
$$

provided that $\Phi_{i}$ is invertible.

Analogously, the future dynamics of individual $i$ and its neighbors $j \in \mathcal{N}(i)^{1}$ can be iterated as follows

$$
\begin{aligned}
& Z_{i}(k+m)=A_{i}^{m} Z_{i}(k), \\
& x_{j}(k+m)=B_{j, i} A_{i}^{m-1} Z_{i}(k),
\end{aligned}
$$

where

$$
\begin{aligned}
& A_{i} \triangleq\left[\begin{array}{c}
B_{i, i} \\
I_{N-1} \quad \mathbf{0}_{(N-1) \times 1}
\end{array}\right] \\
& j \in \mathcal{N}(i) \cup i ; m=1, \ldots, H_{p},
\end{aligned}
$$

and $H_{p}$ is the prediction horizon. Therefore, for an arbitrary individual $i$, provided that the constant parameters 12

${ }^{1} \mathcal{N}(i)$ denotes the set of neighbors of $i$. More precisely, we say that a node $j$ is a neighbor of a node $i$, denoted by $j \in \mathcal{N}(i)$ if, and only if, the corresponding element of the associated adjacency matrix $a_{i j} \neq 0$. 
$B_{j, i}(j \in \mathcal{N}(i) \cup i)$ (see Eqs. (20) and (23)) can be appropriately estimated, the future states of itself and its neighbors can be effectively predicted solely using the historical local state sequences $Z_{j}(k)(j \in \mathcal{N}(i) \cup i)$ observable by individual $i$.

Comparing Eq. (22) with Eq. (16), we note that "current global information" including $P_{\epsilon}$ and $x(k)$ is no longer required for future state prediction as this information can be recovered from "historical local observations" once the local correlation information $B_{j, i}$ has been satisfactorily estimated. In this sense, 'historical local information is equivalent to current global information'.

Estimation of $B_{j, i}$ can be realized provided that each individual possesses a memory of sufficient capacity, allowing it to store the length- $N$ historical state sequences of $x_{i}(k)$ and $x_{j}(k)$. More precisely, since at time step $k$, individual $i$ has already stored $x_{j}(k-n)$ and $Z_{i}(k-n-1)(j \in \mathcal{N}(i) \cup i, n=0,1, \ldots, N-1) \quad$ into its memory, we can use

$$
x_{j}(k-n)=B_{j, i} Z_{i}(k-n-1)
$$

and perform a least square estimation (LSE) [45] to obtain the estimates $\hat{B}_{j, i}$ of the row vectors $B_{j, i}$.

Furthermore, in natural flocks/swarms, it frequently happens that some individuals escape the neighborhood of an individual and enter the neighborhood of others, or that some new individuals join the group while some others leave it, making the network topology change continuously. Fortunately, if the topology modification rate is not too high, the proposed decentralized prediction remains feasible. Indeed, since the prediction is based on the historical information sequence of the last $2 \mathrm{~N}$ steps before current time $k$ (see Eq. (24)), and is aimed at the prediction of the future $H_{p}$ steps (see Eq. (22)), the decentralized predictive protocol (24) remains valid provided that the topology remains constant during $2 N+H_{p}$ steps. Thus, the upper bound of the topology changing rate is $1 /\left(2 N+H_{p}\right)$.

The decentralized predictive protocol (24) is illustrated on the 5-node network shown in Figure 11, in which the topology is changed from Figure 11(a) to Figure 11(b) at the 47th step. Numerical simulations show that, before the topology switch the prediction error $e_{p, i}(k)$ remains small (less than $10^{-7}$ ). Note that the prediction error of node $i$ is defined as $e_{p, i}(k+m)=\left\|x_{i}(k+m)-\hat{x}_{i}(k+m)\right\|_{2}$ $\left(m=1, \ldots, H_{p}\right)$ with $\hat{x}_{i}(k+m)=e_{i} \hat{A}_{i}^{m} Z_{i}(k)$ and $\hat{A}_{i}$ being the least square estimation of $A_{i}$ given in Eq. (23). When the topology switches, the prediction error $e_{p, i}(k)$ rises sharply to approach 0.1 and then begins to oscillate as a result of the transient adaptive process of the prediction. ${ }^{13}$ After less than $N$ steps, $e_{p, i}(k)$ settles down to a level lower than $10^{-7}$. In this way, both the prediction accuracy and the adaptive capability for topology variations have been illustrated through simulation.

\section{B. Decentralized Predictive Protocol}

Since we have established that future state prediction can be obtained from historical local observations, we can introduce a decentralized predictive consensus protocol for general networks. For concision, we ignore the identification error in the following context and deem that $\hat{B}_{j, i}=B_{j, i}(j \in \mathcal{N}(i) \cup i)$.

Similar to the modification proposed in Eq. (10), we add a prediction control input to the routine dynamics given in (21) for $m=1$ :

$$
\begin{aligned}
& Z_{i}(k+1)=A_{i} Z_{i}(k)+e_{1}^{T} v_{i}(k), \\
& x_{i}(k+1)=e_{1} A_{i} Z_{i}(k)+v_{i}(k), \\
& x_{j}(k+1)=B_{j, i} Z_{i}(k),
\end{aligned}
$$

where $v_{i}(k)$ is an additional term representing the MPC action. Using the predictive protocol (25)-(27), the future states of agent $i$ can be predicted based on the currently available historical state sequence $Z_{i}(k)$ as follows:

$$
\begin{aligned}
& X_{i}(k+1)=P_{Z_{i}} Z_{i}(k)+P_{U_{i}} U_{i}(k), \\
& X_{j}(k+1)=P_{Z_{j}} Z_{i}(k)+P_{U_{j}} U_{i}(k)
\end{aligned}
$$

with

$$
\begin{aligned}
X_{i}(k+1) & =\left[x_{i}(k+1), \ldots, x_{i}\left(k+H_{p}\right)\right]^{T}, X_{j}(k+1) \\
& =\left[x_{j}(k+1), \ldots, x_{j}\left(k+H_{p}\right)\right]^{T}, U_{i}(k) \\
& =\left[v_{i}(k), \ldots, v_{i}\left(k+H_{u}-1\right)\right]^{T}, \\
P_{Z_{i}} & =\left[\left(e_{1} A_{i}\right)^{T}, \ldots,\left(e_{1} A_{i}^{H_{p}}\right)^{T}\right]_{H_{p} \times N}^{T}, \\
P_{Z_{j}} & =\left[B_{j, i}^{T},\left(B_{j, i} A_{i}\right)^{T}, \ldots,\left(B_{j, i} A_{i}^{H_{p}-1}\right)^{T}\right]_{H_{p} \times N}^{T},
\end{aligned}
$$

$P_{U_{i}}$ and $P_{U_{j}}$ given in Appendix B. Here, $H_{u} \leq H_{p}$ denotes the control horizon, i.e., the length of the predicted control sequence.

As illustrated in Figure 12, the natural interpretation of the present decentralized MPC protocol can be described as follows. Compared with the centralized predictive protocol given in Figure 8, each individual observes and stores the past trajectories of itself and its neighbors, based on which it computes least square estimates of its neighbors' dynamics $\left(\hat{B}_{i, j}\right.$ and $\left.\hat{A}_{i}\right)$. Upon obtaining these estimations, each individual predicts the future states of its neighbors using Eqs. (28) and (29). Finally, it computes 3 some control signal aiming at minimizing the state difference (or disagreement) in its neighborhood over the interval $\left[k+1, k+H_{p}\right]$. 
Bearing such a picture in mind, we represent the state difference between individual $i$ and its neighbors by a vector $\Delta \mathbb{X}_{i}(k+1)=\operatorname{row}\left\{\Delta X_{r, s}(k+1) \mid r, s \in \mathcal{N}(i) \cup i\right.$ and $r>s\}$ where $\Delta X_{r, s}(k+1)=X_{r}(k+1)-X_{s}(k+1)$ and the notation $y=\operatorname{row}\left\{y_{i}\right\}(i=1, \ldots, n)$ means that $y$ is composed of rows of $y_{i}$, i.e., $y=\operatorname{row}\left\{y_{i}\right\} \Leftrightarrow y=\left[y_{1}^{T}, \ldots, y_{n}^{T}\right]^{T}$. For instance, as shown in Figure 11(b), individual 2 has two neighbors (numbered 1 and 3), therefore $\Delta \mathbb{X}_{2}(k+1)=\left[\left(X_{2}(k+1)-X_{1}(k+1)\right)^{T},\left(X_{3}(k+1)-X_{2}\right.\right.$ $\left.(k+1))^{T},\left(X_{3}(k+1)-X_{1}(k+1)\right)^{T}\right]^{T}$.

It then follows from Eqs. (28) and (29) that

$$
\Delta \mathbb{X}_{i}(k+1)=P_{\mathbb{Z}_{i}} Z_{i}(k)+P_{\mathbb{U}_{i}} U_{i}(k)
$$

with $\quad r, s \in \mathcal{N}(i) \cup i$ and $r>s, \quad P_{\mathbb{Z}_{i}}=\operatorname{row}\left\{P_{Z_{r}}-P_{Z_{s}}\right\} \quad$ and $P_{\mathbb{U}_{i}}=\operatorname{row}\left\{P_{U r}-P_{U s}\right\}$.

To solve the consensus problem, we first set the moving horizon optimization index that defines the decentralized MPC consensus problem as follows:

$$
J_{i}(k)=\left\|\Delta \mathbb{X}_{i}(k+1)\right\|_{Q_{i}}^{2}+\left\|U_{i}(k)\right\|_{R_{i}}^{2},
$$

where $Q_{i}$ and $R_{i}$ are compatible real, symmetric, positive definite weighting matrices. In general, the weighting matrices can be set as

$$
Q_{i}=q I_{H_{p} N_{i}\left(N_{i}+1\right) / 2}(q>0) \quad \text { and } \quad R_{i}=I_{H_{u}}
$$

where $N_{i}$ is the number of neighbors of individual $i$. In the optimization index (31), the first term penalizes the disagreement among the neighborhood of agent $i$ over the future $H_{p}$ steps, while the second penalizes the additional MPC control energy $U_{i}(k)$. In order to minimize (31), we compute $\partial J_{i}(k) / \partial U_{i}(k)=0$, and consequently obtain the optimal MPC action as:

$$
v_{i}(k)=P_{M P C, i} Z_{i}(k)
$$

with

$$
\begin{aligned}
P_{M P C, i}= & -[1,0, \ldots, 0]_{1 \times H_{u}} \\
& \cdot\left(P_{\mathbb{U}_{i}}^{T} Q_{i} P_{\mathbb{U}_{i}}+R_{i}\right)^{-1} P_{\mathbb{U}_{i}}^{T} Q_{i} P_{\mathbb{Z}_{i}} .
\end{aligned}
$$

Note that, in this decentralized MPC, the control law $v_{i}(k)$ is calculated and implemented by each individual, which is totally different from the centralized MPC given by Eq. (15).

Substituting Eq. (33) into Eq. (25) yields

$$
Z(k+1)=W Z(k)
$$

with $W=\operatorname{diag}\left\{A_{i}+e_{1}^{T} P_{\mathrm{MPC}, \mathrm{i}}\right\}_{i=1, \ldots, N} \quad$ and $\quad Z(k)=$ $\left[Z_{1}(k)^{T}, \ldots, Z_{N}(k)^{T}\right]^{T}$.
Based on the above discussion, the decentralized MPC algorithm can be divided into two stages:

Stage 1 (Pure identification stage): In the first $2 N$ steps, the LSE method (24) is implemented to yield initial estimates $\hat{A}_{i}$ and $\hat{B}_{j, i}$ of the neighbors' dynamics;

Stage 2 (identification and control stage): From the $2 N+1^{\text {th }}$ step, the MPC is kicked off. At each step $k$, the matrices $P_{\mathrm{MPC}, \mathrm{i}}$ are calculated according to Eq. (34) using the estimates $\hat{A}_{i}, \hat{B}_{j, i}$. The MPC term $v(k)$ is then computed according to Eq. (33) and introduced into each node as shown in Eq. (26). Finally, the LSE method (24) is iterated to yield the updated estimates $\hat{A}_{i}$ and $\hat{B}_{j, i}$.

To support the above proposed decentralized MPC, it is necessary to give some sufficient conditions guaranteeing convergence towards average-consensus. To this end, we first provide a lemma on average-consensus.

Lemma 1: [44] For any matrix $W \in \mathbb{R}^{M \times M}$, the equation

$$
\lim _{k \rightarrow \infty} W^{k}=\mathbf{1}_{M} \mathbf{1}_{M}^{T} / M
$$

with $\mathbf{1}_{M}=[1, \ldots, 1]_{M \times 1}^{T}$ holds if and only if assumptions $A 1$ and $A 2$ hold:

A1: The following holds for $W$

$$
W \mathbf{1}_{M}=W^{T} \mathbf{1}_{M}=\mathbf{1}_{M}
$$

A2: The matrix $W$ has a simple eigenvalue at 1 and all its other eigenvalues inside the open unit circle.

Based on Lemma 1, we give hereafter the necessary and sufficient conditions guaranteeing average-consensus for the proposed decentralized MPC protocol.

Theorem 1: For an $N$-node balanced network with dynamics determined by (35), provided that $\Phi_{i}, i=1, \ldots, N$ (see Eq. (19)) are invertible and $\hat{B}_{j, i}=B_{j, i}$ (see Eq. (20)), then the system state $x(k)$ asymptotically converges to the average-consensus value $\bar{x}(0) \mathbf{1}_{N}$ with $\bar{x}(0) \triangleq 1 / N \sum_{i=1}^{N} x_{i}(0)$ if and only if assumptions $\boldsymbol{A 3}$ and $\boldsymbol{A 2}$ hold

A3: The following equality holds

$$
\mathbf{1}_{N}^{T} \cdot\left(A_{i}+e_{1}^{T} P_{\mathrm{MPC}, \mathrm{i}}\right)=\mathbf{1}_{N}^{T}(i=1, \ldots, N) .
$$

The assumption $\boldsymbol{A 3}$ can be interpreted as that the internal prediction-related effects are counteracted. It can 14 be numerically checked that in the 3-dimensional space spanned by the parameters $H_{u}, H_{p}$, and $q$, there is a fairly large region in which $\boldsymbol{A 3}$ is fulfilled (such as the region 
corresponding to the common parameter settings $H_{u} \in[1,10], H_{p} \in\left[H_{u}, 10\right]$ and $\left.q \in[0.01,10]\right)$.

Furthermore, similar to the nominal MPC's performance shown in Figure 10, the eigenvalues distribution of $W$ (see Eq. (35)) is always much smaller and closer to the origin than the one of $P_{\epsilon}$, which explains the overall higher consensus speed of the decentralized MPC protocol. More significantly, when $\epsilon$ is increased beyond the threshold $1 / d_{\max }$ (see Figure 14(b) where $\epsilon=2$ ), some of the eigenvalues of $P_{\epsilon}$ start escaping the unit circle, making the disagreement function diverge, whereas all the eigenvalues of $W$ remain inside the unit circle (except one that is always located at 1), which ensures its convergence. Clearly, from the mathematical point of view, the effect of predictive mechanism is to drive the escaping eigenvalues towards the origin. One may notice that, compared with nominal MPC, the eigenvalue distribution of decentralized MPC is less compact around the origin. This is due to the parameter estimation error which inevitably leads to slight inaccuracies in the state predictions.

To vividly illustrate the virtues of the decentralized predictive consensus protocol, we present some simulation results comparing the convergence speeds obtained using the routine protocol given in Eq. (9) and the proposed predictive protocol given in Eq. (35) on the 10-node network given in Figure 13(a). Once again, since the objective is to reach average-consensus, the instantaneous disagreement index is set as $D(k)=\left\|x(k)-\mathbf{1}_{N} \bar{x}(0)\right\|_{2}^{2}$.

In the convergent case of the routine protocol, e.g., for $\epsilon \leq 1 / d_{\max }$, the decentralized MPC consensus protocol (35) yields an increase in the convergence speed towards average-consensus (by a factor of 3 approximately), as shown in Figure 15(a). Furthermore, even when the routine convergence conditions are violated, e.g., $\epsilon>1 / d_{\max }$, it is observed in Figures 15(b) and 15(c) that the decentralized MPC consensus protocol still allows asymptotic convergence to average-consensus. Thus, the range of sampling period $\epsilon$ leading to asymptotic convergence towards consensus is expanded using the decentralized predictive mechanism. Interestingly, one may ${ }^{15}$ notice the slight oscillations of the MPC consensus protocol's $D(k)$ curve in Figures 15(a) and (b), which root in the online identification and adaptation processes performed by each node.

Finally, to demonstrate the decentralized MPC's superiority in handling network topology switches, we compare the consensus performances of the MPC and routine protocols in Figure 16, when the topology is switched from Figure 13(a) to Figure 13(b) at the 43rd running step. For the routine protocol, since $\{0.48,0.49\} \in[1 / 3,1 / 2](1 / 2$ and $1 / 3$ are the $1 / d_{\max }$ values of the topologies considered in Figure 11(a) and (b), respectively), the averageconsensus tendency is broken shortly after the network topology switch. On the contrary, the average-consensus

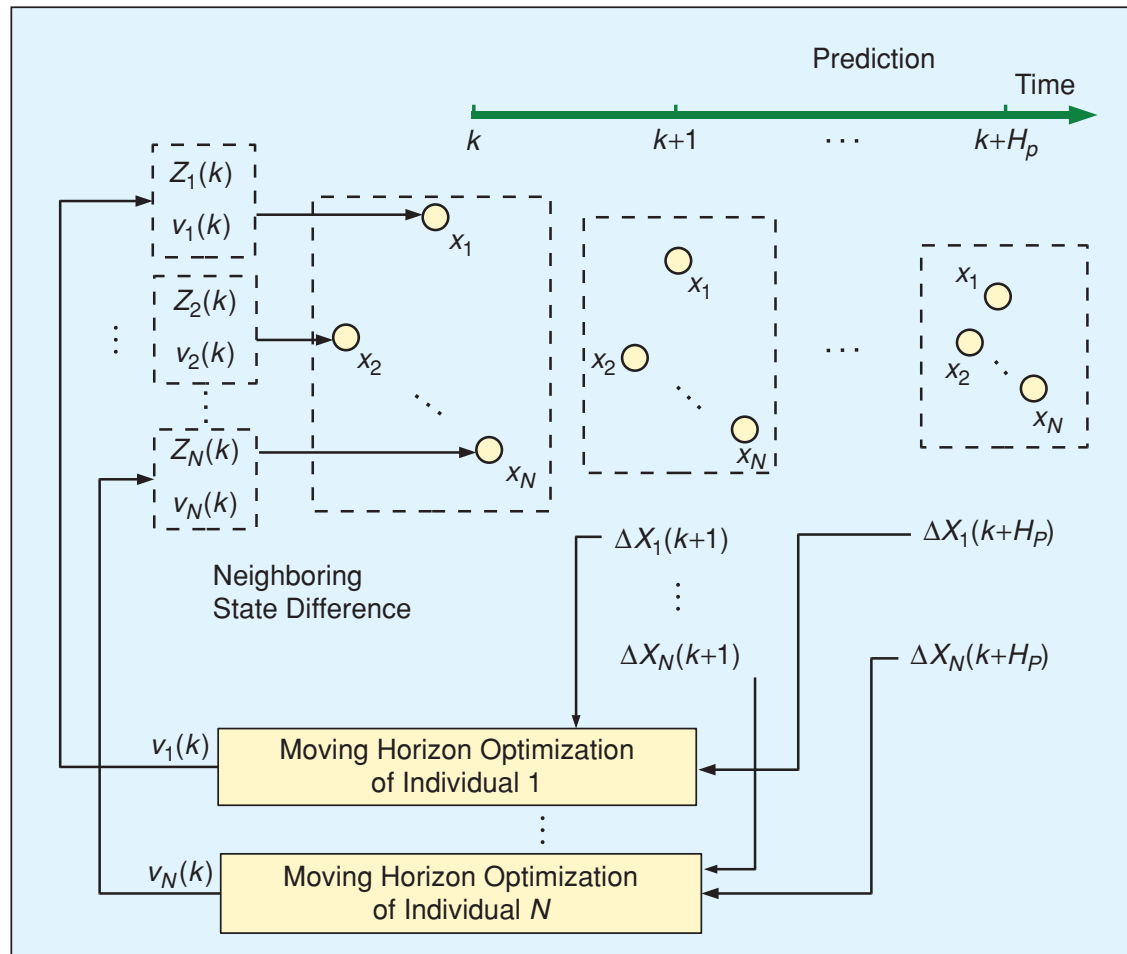

Figure 12. Decentralized MPC protocol. tendency holds all along for the decentralized MPC consensus protocol since it can adapt to the topology changes and use the additional MPC term (see Eq. (26)) to steer each node towards its neighbors.

\section{Conclusion}

To extract the role of predictive mechanisms that may extensively exist in abundant natural bio-groups, we surveyed some recent investigations on collective group behaviors, and designed three predictive mechanisms both in centralized and decentralized manners for flocks with or without leaders. By using mathematical analysis and numerical simulations, we illustrated the advantages 15 of such predictive protocols for both the exploration of emergent behaviors and the design of autonomous and reliable consensus networks. 


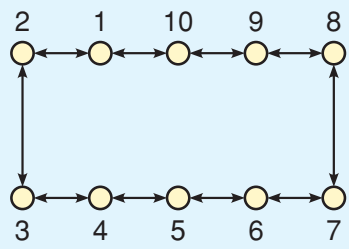

(a)

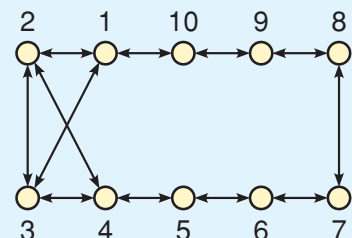

(b)

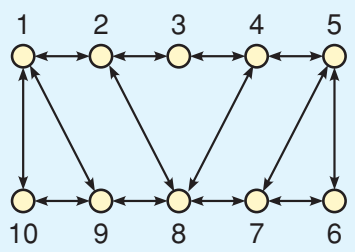

(c)

Figure 13. Network topologies used.

For flocks with leaders, we designed a small-world predictive protocol for two mainstream models, namely the $\mathrm{A} / \mathrm{R}$ and Vicsek models. In this scenario, the predictive capability is incorporated into the long-range connections from the leader to the pseudo-leaders. Simulation results led to the following conclusions: 1 ) owing to the predictive capability of the pseudo-leaders, the group becomes more cohesive to the leader and the flock formation is more stable; 2) predictive capability can compensate for the need of costly long-range connections.

For flocks without leaders, which is a more general case in nature, we incorporated a predictive term into the routine consensus law, with the aim of minimizing the future state difference between any pair of individuals. Both numerical simulations and mathematical analysis were provided to show the advantages of such centralized MPC protocols. In particular, we showed that 1 ) even short-term predition leads to a significant increase in convergence speed; 2) the sampling period range guaranteeing convergence can be sharply increased, giving the MPC protocol the potential to effectively save communication energy.

In nature, each individual typically lacks global knowledge of the network state and topology and can merely plan its motion based on observable local information. In the third part, we designed a decentralized MPC protocol, for which computation is distributed to each individual. To guarantee the feasibility of such a decentralized protocol, we proved that each individual can estimate the future states of its neighbors by solely using the historical observable local information sequence. In this sense, 'historical local' is equivalent to 'current global.' Once the neighbors' states have been predicted, an MPC consensus protocol similar to the centralized one can be easily designed. Both numerical simulations and mathematical analysis showed that, compared with the above centralized predictive protocol, the performance improvements in the decentralized scenario are slightly reduced while the above-mentioned two virtues of predictive mechanism still hold. More significantly, this decentralized predictive protocol is more realistic and clearly more useful in the design of industrial applications.

For natural science, the contribution of this work lies in its ability to explain why individuals of biological flocks/swarms like fireflies and deep-sea fish do not

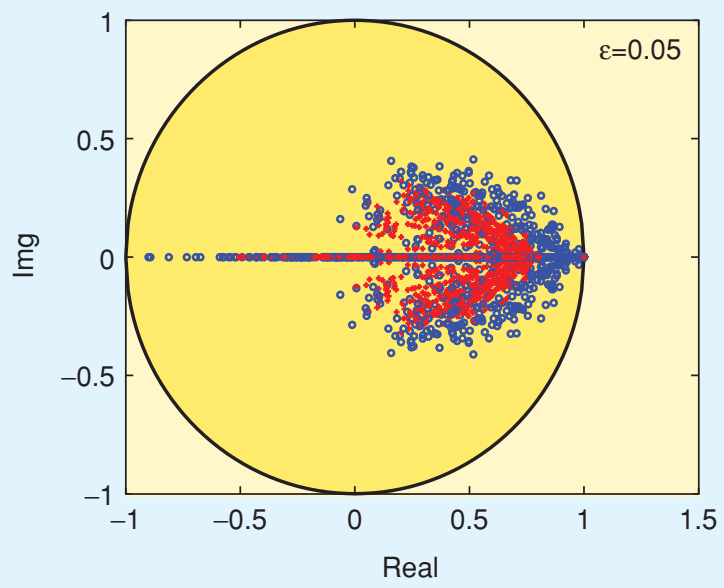

(a)

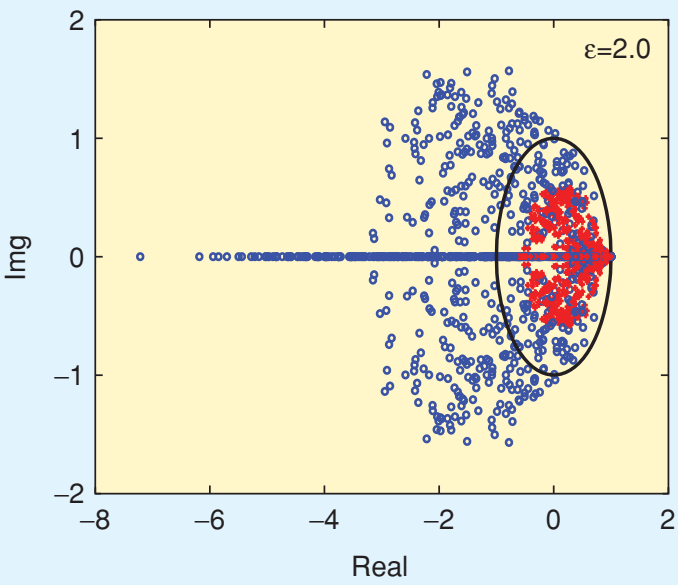

(b)

Figure 14. (Color online) Eigenvalue distributions for different $\epsilon$. Blue $(\bigcirc)$ and red $(+)$ represent the eigenvalues of $P_{\epsilon}$ and $W$ (see Eq. (35)) over 100 runs, respectively. The simulations are implemented on the balanced network with topology given in Figure 13(c). The eigenvalue distribution of $W$ correspond to snapshot $\$$ at the 50 th running step. The black circle denotes the unit circle in the complex plane. Here, $H_{p}=7, H_{u}=1, q=0.015$, and each entry $I_{i j}(j \neq i$, and $(i, j) \in \mathcal{E})$ is chosen randomly in $[-1,0)$ such that the resulting network is balanced. The associated values of $d_{\max }$ lie in $[0.9,2.7]$. 


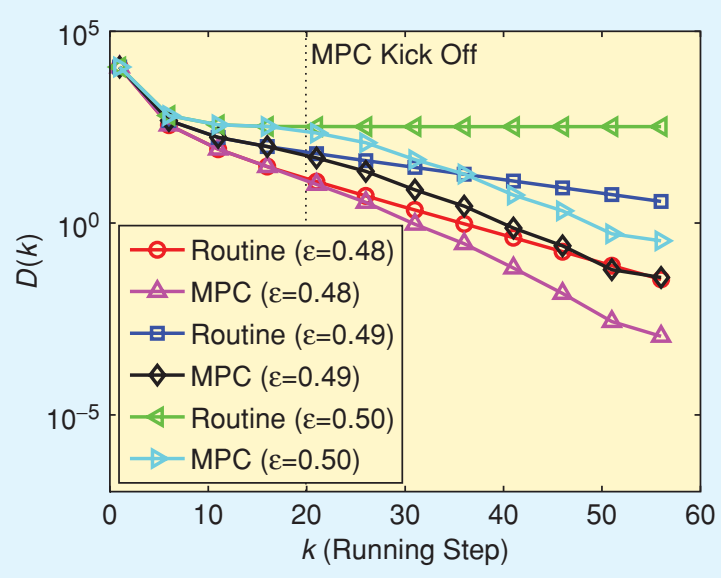

(a)

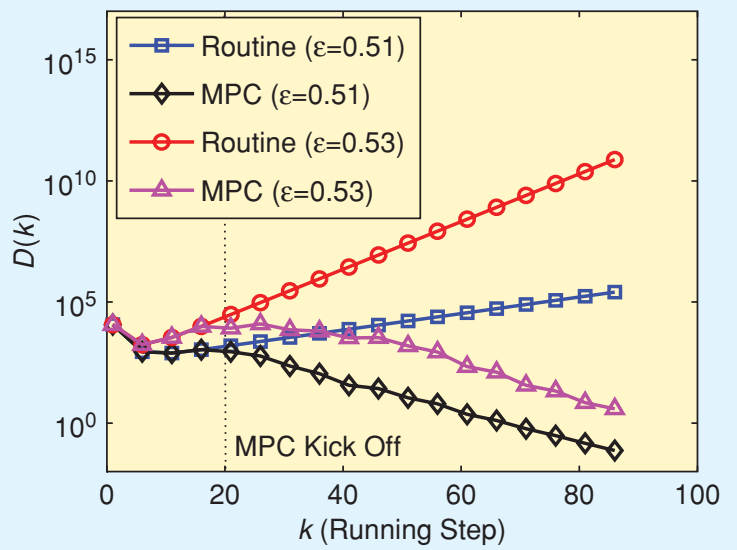

(b)

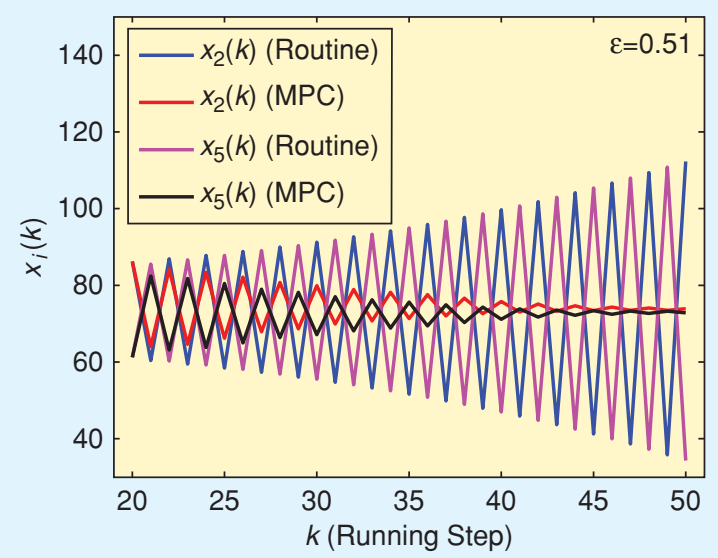

(c)

Figure 15. (Color online) Consensus performance comparison of the routine and predictive protocols on the balanced network represented in Figure 13(a) for which $d_{\max }=2$. (a) Time evolution of the instantaneous disagreement $D(k)$ for $\epsilon \leq 1 / d_{\max }$ (convergent case of the routine protocol); (b) Time evolution of the instantaneous disagreement $D(k)$ for $\epsilon>1 / d_{\max }$ (divergent case of the routine protocol); (c) state evolution for $\epsilon=0.51$. Here, $a_{i j}=1$ if $(i, j) \in \mathcal{E}$, otherwise $a_{i j}=0$, the initial state $x_{i}(0)$ is selected randomly in $[0,150], H_{p}=7, H_{u}=1, \quad N=10$, $q=0.015$. Each point is an average over 500 independent runs.

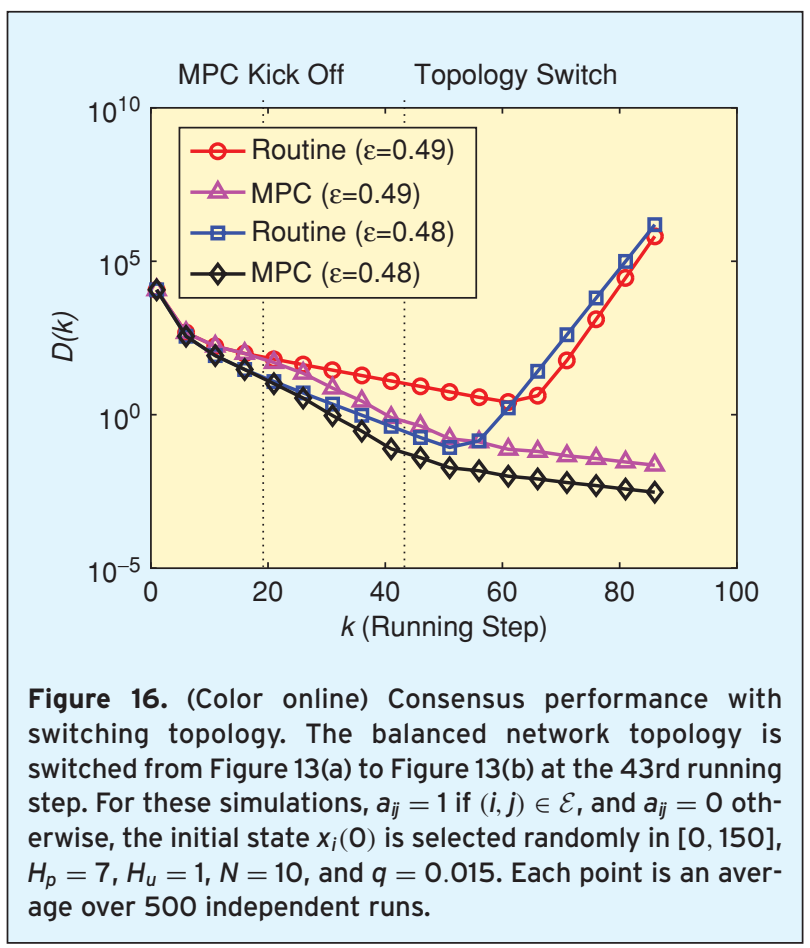

communicate very frequently all along but just now and then during the whole dynamic process. From the industrial application point of view, the value of this work is twofold. The consensus performance is significantly enhanced while the communication energy or cost is effectively reduced. All these virtues are only at the cost of giving the agents the capabilities of storing past states and making predictions. This work is just a first attempt to reveal the role of collective predictive mechanism for natural flocks/swarms and thereby improving the performances of industrial multi-agent systems' collective behaviors. We believe that numerous research issues remain open in the ongoing efforts to design predictive protocols that are efficient, robust and scalable to large-sized systems. We also expect the coming years to embrace an intensive development about the collective behavior coordination via predictive mechanism and the relevant applications in multi-agent industrial systems.

\section{Acknowledgments}

The authors would like to thank Mr. Cheng Zhao for his assistance in the manuscript preparation. H. T. Zhang acknowledges the support of the National Natural Science Foundation of China (NNSFC) under Grant No. 60704041, and the Research Fund for the Doctoral Program of Higher Education (RFDP) under Grant No. 20070487090. G.-B. Stan acknowledges the support of the U.K. Engineering and ${ }^{17}$ Physical Sciences Research Council (EPSRC) under the grant EP/E02761X/1. T. Zhou acknowledges the support of NNSFC under Grant No. 10635040. 


\section{Appendix}

\section{A. Prediction matrix for centralized MPC}

$$
P_{U}=\left[\begin{array}{cccc}
I_{N} & & & \\
P_{\epsilon} & I_{N} & & \\
\vdots & \vdots & \ddots & \\
P_{\epsilon}^{H_{p}-1} & P_{\epsilon}^{H_{p}-2} & \ldots & I_{N}
\end{array}\right]
$$

\section{B. Prediction matrices for decentralized MPC}

$$
\begin{aligned}
& P_{U_{i}}=
\end{aligned}
$$

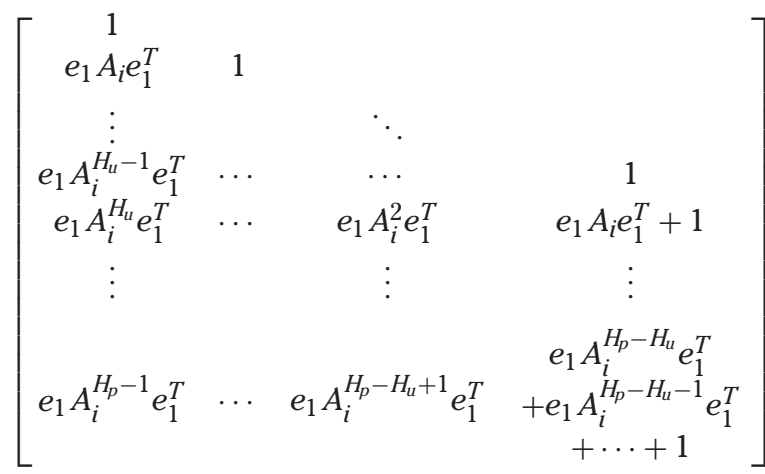

$$
\begin{aligned}
& P_{U_{j}}= \\
& {\left[\begin{array}{cccc}
0 & & & \\
B_{j, i} e_{1}^{T} & 0 & & \\
\vdots & & \ddots & 0 \\
B_{j, i} A_{i}^{\dot{H}_{u}-2} e_{1}^{T} & \ldots & \cdots & B_{j, i} e_{1}^{T} \\
B_{j, i} A_{i}^{H_{u}-1} e_{1}^{T} & \ldots & B_{j, i} A_{i} e_{1}^{T} & \vdots \\
\vdots & & \vdots & B_{j, i} A_{i}^{H_{p}-H_{u}-1} e_{1}^{T} \\
& & & +B_{j, i} A_{i}^{H_{p}-H_{u}-2} e_{1}^{T} \\
B_{j, i} A_{i}^{H_{p}-2} e_{1}^{T} & \ldots & B_{j, i} A_{i}^{H_{p}-H_{u}} e_{1}^{T} & +\cdots+B_{j, i} e_{1}^{T}
\end{array}\right]}
\end{aligned}
$$

\section{References}

[1] D. Helbing, I. Farkas, and T. Vicsek, "Simulating dynamical features of escape panic," Nature, vol. 407, pp. 487-490, 2000.

[2] T. Vicsek, "A question of scale," Nature, vol. 411, pp. 421-421, 2001.

[3] E.O. Budrene and H. Berg, "Dynamics of formation of symmetrical patterns by chemotactic bacteria," Nature, vol. 376, pp. 49-53, 1995.

[4] S. Martínez, J. Cortés, and F. Bullo, "Motion coordination with distributed information," IEEE Control Systems Magzine, vol. 27, pp. 75-88, 2007.

[5] S. Nadis, "Complex systems: All together now," Nature, vol. 421, pp. 780-782, 2003.

[6] Z. Néda, E. Ravasz, V. Brechet, T. Vicsek, and A.L. Barabási, "Self-organizing processes: The sound of many hands clapping," Nature, vol. 403, pp. 849-850, 2000

[7] M.B. Miller and B.L. Bassler, "Quorum sensing in bacteria," Ann. Rev. Microbiol., vol. 55, pp. 165-199, 2001.

[8] A. Okubo, "Dynamical aspects of animal grouping: swarms, schools, flocks, and herds," Adv. Biophys., vol. 22, pp. 1-94, 1986

[9] R. Olfati-Saber and R. Murray, "Consensus problems in networks of agents with switching topology and time-delays," IEEE Trans. Automat. Contr., vol. 18 49, pp. 1520-1533, 2004.

[10] W. Ren, R.W. Beard, and E.M. Arkins, "Information consensus in multivehicle cooperative control,” IEEE Control System Magazine, vol. 71, pp. 71-82, 2004.
[11] I.F. Akyildiz, W. Su, Y. Sankarasubramaniam, and E. Cayirci, "Wireless sensor networks: a survey," Computers Network, vol. 38, pp. 393-422, 2002.

[12] P. Ogren, E. Fiorelli, and N.E. Leonard, "Cooperative control of mobile sensor networks: Adaptive gradient climbing in a distributed environment," IEEE Trans. Automat. Contr., vol. 49, pp. 1292-1302, 2004.

[13] T. Arai, E. Pagello, and L.E. Parker, "Guest editorial advances in multirobot systems," IEEE Trans. Robotics and Automation, vol. 18, pp. 655-661, 2002.

[14] M. Fiedler, "Algebraic connectivity of graphs," Czechoslovak Mathematical Journal, vol. 23, pp. 660-670, 1973

[15] R. Olfati-Saber, "Ultrafast consensus in small-world networks," in Proc. American Control Conference, June 2005, pp. 2371-2378.

[16] S.H. Strogatz, "Exploring complex networks," Nature, vol. 410, pp. 268-275, 2001.

[17] W. Yang, L. Cao, X.F. Wang, and X. Li, "Consensus in a heterogeneous influence network," Phys. Rev. E, vol. 74, 037101, 2006.

[18] W. Li and X.F. Wang, "Adaptive velocity strategy for swarm aggregation," Phys. Rev. E, vol. 75, 021917, 2007.

[19] W. Li, H.T. Zhang, M.Z.Q. Chen, and T. Zhou, "Singularities and symmetry breaking in swarms," Phys. Rev. E, vol. 77, 021920, 2008.

[20] J. Zhang, Y. Zhao, B. Tian, L. Peng, H.T. Zhang, B.H. Wang, and T. Zhou, "Accelerating consensus of self-driven swarm via adaptive speed," arXiv: 0711.3896, 2007.

[21] C.W. Reynolds, "Flocks, herds and schools: A distributive behavioral model," Computer Graphics, vol. 21, pp. 25-34, 1987.

[22] V. Gazi and K.M. Passino, "Stability analysis of swarms," IEEE Trans. Automat. Contr., vol. 48, pp. 692-697, 2003.

[23] V. Gazi and K.M. Passino, "Stability analysis of social foraging swarms," IEEE Trans. Systems, Man, and Cybernetics, vol. 34, pp. 539-557, 2004.

[24] H. Shi, L. Wang, and T. Chu, "Virtual leader approach to coordinated control of multiple mobile agents with asymmetric interactions," Physica D, vol. 213, pp. 51-65, 2006.

[25] I.D. Couzin, J. Krause, R. James, G.D. Ruxton, and N.R. Franks, "Collective memory and spatial sorting in animal groups," J. Theor. Biol., vol. 218, pp. $1-11,2002$.

[26] C. Zimmer, "From ants to people, an instinct to swarm," The New York Times, Nov. 13, 2007.

[27] T. Vicsek, A. Czirók, E. Ben-Jacob, I. Cohen, and O. Shochet, "Novel type of phase transition in a system of self-driven particles," Phys. Rev. Lett. vol. 75 , pp. 1226-1229, 1995

[28] A. Jadbabaie, J. Lin, and A.S. Morse, "Coordination of groups of mobile autonomous agents using nearest neighbor rules," IEEE Trans. Autom. Contr., vol. 48, pp. 988-1001, 2003

[29] E.F. Woods, "Electronic prediction of swarming in bees," Nature, vol. 184, pp. 842-844, 1959.

[30] P.R. Montague, P. Dayan, C. Person, and T.J. Sejnowski, "Bee foraging in uncertain environments using predictive hebbian learning," Nature, vol. 377, pp. 725-728, 1995.

[31] J.A. Gottfried, J.O. Doherty, and R.J. Dolan, "Encoding predictive reward value in human amygdala and orbitofrontal cortex," Science, vol. 301, pp. 1104-1107, 2003.

[32] C. Summerfield, T. Egner, M. Greene, E. Koechlin, J. Mangles, and J. Hirsch, "Predictive codes for forthcoming perception in the frontal cortex," Science, vol. 314, pp. 1311-1314, 2006.

[33] D. Melcher, "Predictive remapping of visual features precedes saccadic eye movements," Nature Neuroscience, vol. 10, pp. 903-907, 2007.

[34] D.J. Watts and S.H. Strogatz, "Collective dynamics of small-world networks," Nature, vol. 393, pp. 440-443, 1998.

[35] I.D. Couzin, J. Krause, N.R. Franks, and S.A. Levin, "Effective leadership and decision-making in animal groups on the move," Nature, vol. 433, pp. 513-516, 2005.

[36] S. Janson, M. Middendorf, and M. Beekman, "Honeybee swarms: how do scouts guide a swarm of uninformed bees?," Animal Behaviour, vol. 70, pp. 349-358, 2005.

[37] H.T. Zhang, M.Z.Q. Chen, and T. Zhou, "Predictive protocol of flocks with small-world connection pattern," arXiv 0709.0184, 2007.

8 [38] L. Conradt and T.J. Roper, "Group decision-making in animals," Nature, vol. 421, pp. 155-158, 2003.

[39] J. Halloy, G. Sempo, G. Caprari, C. Rivault, M. Asadpour, F. Tache, I. Saïd, V. Durier, S. Canonge, J.M. Amé, C. Detrain, N. Correll, A. Martinoli, F. Monda- 
da, R. Siegwart, and J.L. Deneubourg, "Social integration of robots into groups of cockroaches to control self-organized choices," Science, vol. 318, pp. 1155-1158, 2007.

[40] J.K. Parrish, S.V. Viscido, and D. Grunbaum, "Self-organized fish schools: an examination of emergent properties," Biol. Bull., vol. 202, pp. 296-305, 2002.

[41] S. Gueron and S.A. Levin, "Self-organization of front patterns in large wildebeest herds," J. Theoretical Biol., vol. 165, pp. 541-552, 1993.

[42] T.D. Seeley and S.C. Buhrman, "Group decision making in swarms of honey bees," Bahav. Ecology Sociobiol., vol. 45, pp. 19-31, 1999.

[43] H.T. Zhang, G.B. Stan, M.Z.Q. Chen, and T. Zhou, "Greatly improving consensus performance via predictive mechanism," arXiv:0709.0172, 2007.

[44] L. Xiao and S. Boyd, "Fast linear iterations for distributed averaging," Systems \& Control Letters, vol. 53, pp. 65-78, 2004.

[45] L. Ljung, System Identification: Theory for the User, Prentice-Hall, Inc. Englewood Cliffs, N.J., 1999.

[46] L. Diambra and A. Plastino, "Maximum entropy, pseudoinverse techniques, and time series predictions with layered networks," Phys. Rev. E, vol. 52, pp. 4557-4560, 1995.

[47] T. Zhou, M. Zhao, and B.H. Wang, "Better synchronizability predicted by crossed double cycle," Phys. Rev. E, vol. 73, 037101, 2006. 